\title{
PLANE WAVE ULTRASOUND IMAGING USING SYNTHETIC APERTURE IMAGE RECONSTRUCTION TECHNIQUES
}

\author{
by \\ Dae-Myoung (Danny) Yang \\ Bachelor of Science in Medical Physics, \\ University of Western Ontario, London, Ontario, Canada, 2013 \\ A thesis
presented to Ryerson University \\ in partial fulfillment of the \\ requirements for the degree of \\ Master of Science \\ in the Program of \\ Biomedical Physics
}

Toronto, Ontario, Canada, 2016

(C) Dae-Myoung Yang, 2016 


\section{AUTHOR'S DECLARATION}

I hereby declare that I am the sole author of this thesis. This is a true copy of the thesis, including any required final revisions, as accepted by my examiners.

I authorize Ryerson University to lend this thesis to other institutions or individuals for the purpose of scholarly research

I further authorize Ryerson University to reproduce this thesis by photocopying or by other means, in total or in part, at the request of other institutions or individuals for the purpose of scholarly research.

I understand that my thesis may be made electronically available to the public.

Dae-Myoung Yang 


\title{
PLANE WAVE ULTRASOUND IMAGING USING SYNTHETIC APERTURE IMAGE RECONSTRUCTION TECHNIQUES
}

\author{
Master of Science 2016 \\ Dae-Myoung Yang \\ Biomedical Physics \\ Ryerson University
}

\begin{abstract}
Ultrasound imaging based on transmitting plane waves (PW) enables ultrafast imaging. Coherent PW compounding ultrasound imaging can reach the image quality of optimal multifocus image. In the image reconstruction, it was assumed that an infinite extent PWs was emitted. In this thesis, we propose a new image reconstruction algorithm - Synthetic-aperture plane-wave (SAPW) imaging - without using this assumption. The SAPW imaging was compared with the PWs imaging in numerical simulations and experimental measurements. The measured RF data in PW imaging was first decoded in the frequency domain using a pseudoinverse algorithm to estimate the RF data Then, SAPW RF data were used to reconstruct images through the standard synthetic transit aperture (STA) method. Main improvements in the image quality of the SAPW imaging in comparison with the PWs imaging are increases in the depth of penetration and the field of view when contrast-to-noise ratio (CNR) was used as a quantitative metric.
\end{abstract}




\section{ACKNOWLEDGEMENTS}

I would like to thank my supervisors Dr. Yuan Xu and Dr. Jahan Tavakkoli for their continuous advices, assistance, patience, encouragement and guidance in this research study.

I would like to thank my supervisory committee members Dr. J. Carl Kumaradas and Dr. Michael C. Kolios for their valuable guidance and feedback.

I would like to thank my defence committee member Dr. Raffi Karshafian for his time, questions and feedback.

Special thanks go to Xu lab members Ping Gong and Ying Li for their assistances.

I acknowledge my family and colleagues for their encouragement and spiritual support.

Last, but not least, I would like to thank God.

This work was supported by the Natural Sciences and Engineering Research Council of Canada (NSERC) Discovery grants that were awarded to Dr. Y. Xu and Dr. J. Tavakkoli. 


\section{TABLE OF CONTENTS}

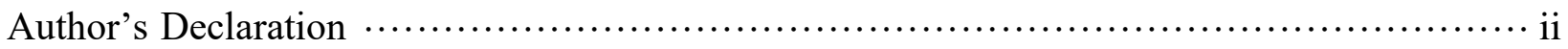

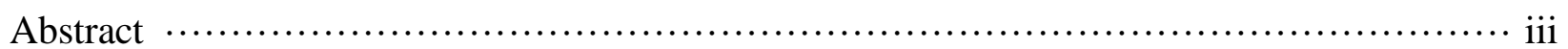

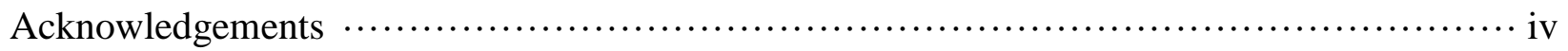

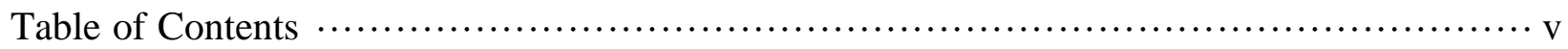

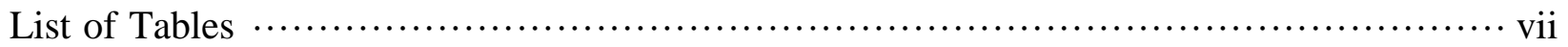

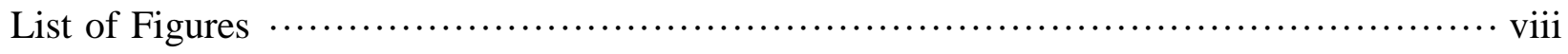

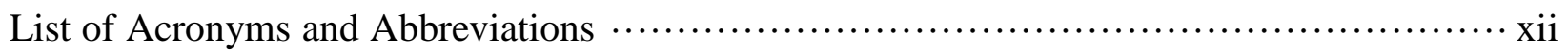

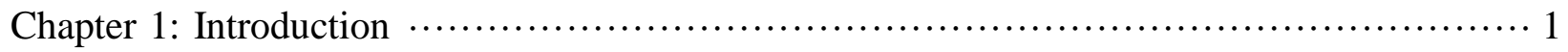

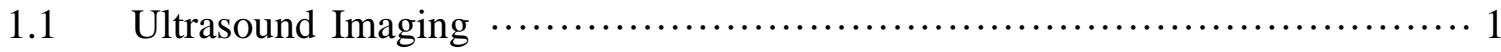

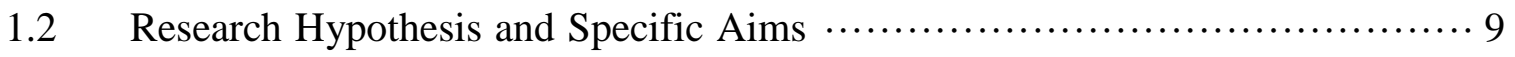

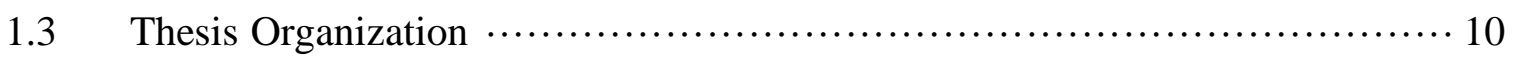

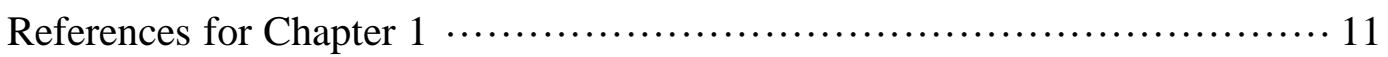

Chapter 2: Plane Wave Ultrasound Imaging Using Synthetic Aperture Image Reconstruction

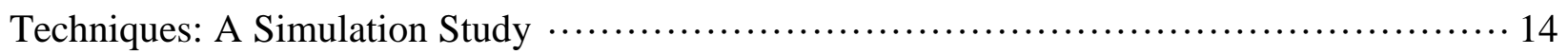

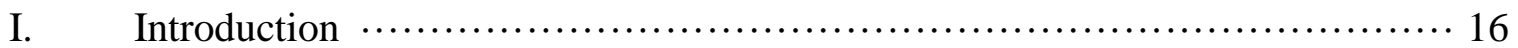

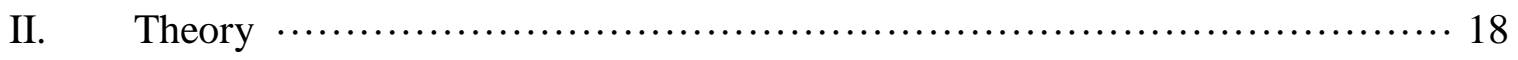

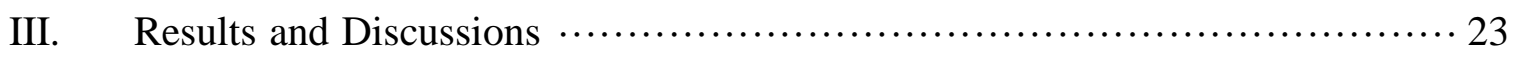

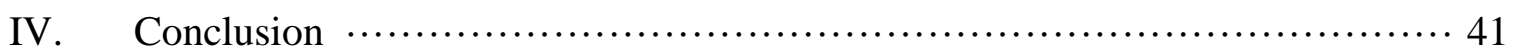

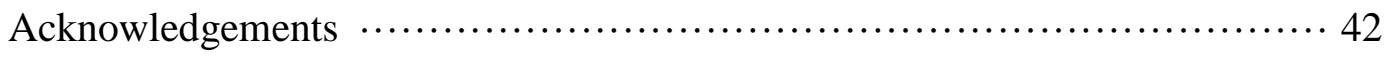

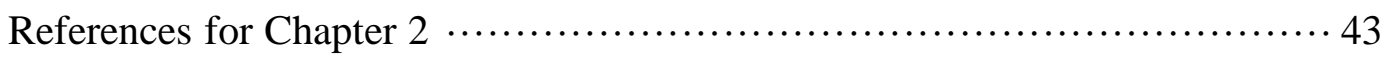

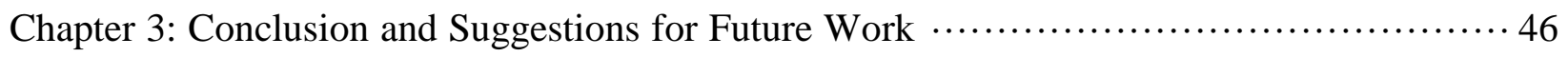

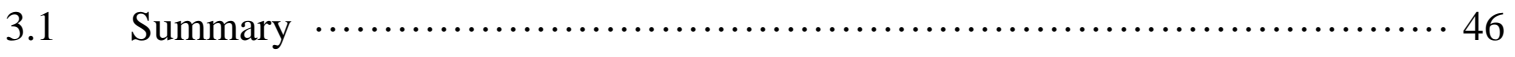




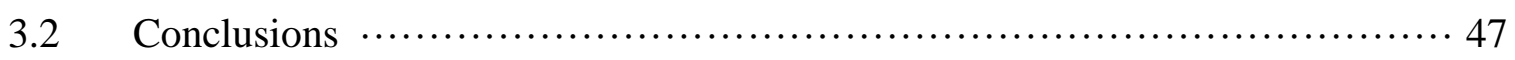

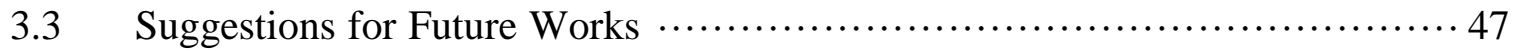

Appendix A: A Preliminary Experimental Validation of the Synthetic-Aperture Plane Wave

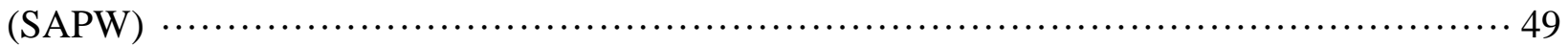

Appendix B: Comparison Between Hadamard Encoded Transmission and Specifically Selected

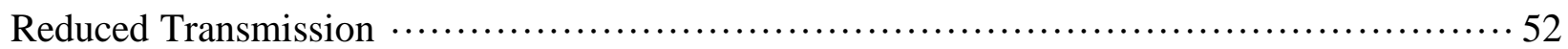

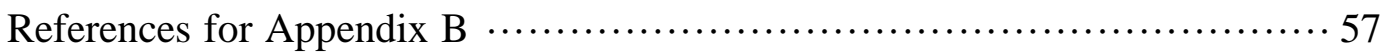




\section{LIST OF TABLES}

Table 1.1: Quantitative comparison for the different methods. The SNR is relative to the PW

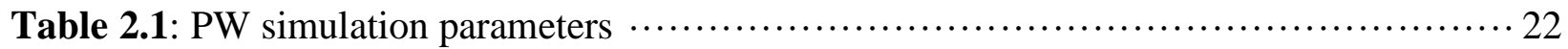

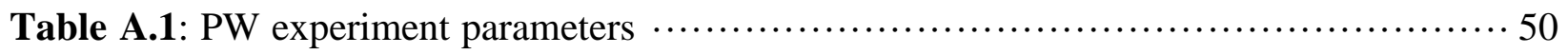

Table A.2: Preliminary experimental quantitative comparison for the different methods $\cdots \cdots 51$ 


\section{LIST OF FIGURES}

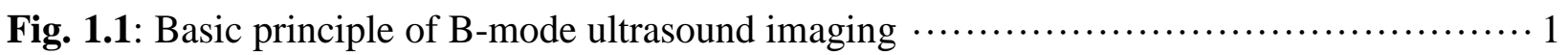

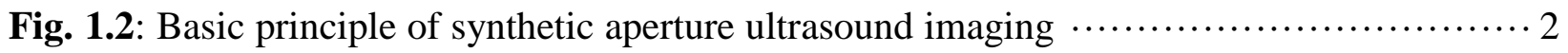

Fig. 1.3: Schematic representation of the single transmit PW method: (a) the ultrasonic array insonifies the medium using a PW transmission without steering, and (b) the backscattered RF

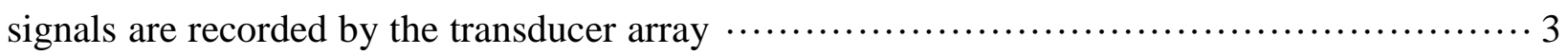

Fig. 1.4: (a) Axis convention, (b) distance to the point $(x, z)$ and back to transducer for a PW, and (c) for a PW of angle $\alpha$ steering

Fig. 1.5: (a) B-mode image obtained using a single PW transmission, (b) Coherent (b) Coherent PW compound using 71 angles separated $0.47^{\circ}$; value given by (2.13). (c) Standard monofocal B-mode image using a single focal depth at $30 \mathrm{~mm}$. (d) Multifocal image using 4 successive focal depths

Fig. 1.6: Images of anechoic objects using different number of PWs. (a) 1 PW, (b) 5 PWs separated $2^{\circ}\left(-4^{\circ},-2^{\circ}, 0^{\circ}, 2^{\circ}, 4^{\circ}\right)$, (c) $21 \mathrm{PWs}$ separated $1^{\circ}$, and (d) $45 \mathrm{PWs}$ separated $0.5^{\circ}$

Fig. 2.1: Region of interest (ROI) in anechoic cyst simulation to estimate CNR. The white box indicates the ROI inside the cyst and black boxes indicates ROI outside the cyst. The mean and standard deviation of pixel values inside and outside the cyst were used to compute CNR as

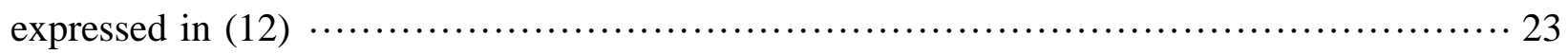

Fig. 2.2: Simulated log-enveloped beamformed PW (left) and SAPW (right) imaging using the optimal configuration of PW in tissue mimicking phantom

Fig. 2.3: CNR comparison between PW (dashed lines) and SAPW (solid lines) of Fig. 2.2 at different depth at the $0 \mathrm{~mm}$ lateral distance (left) and different lateral distance at depth $30 \mathrm{~mm}$ (right) 
Fig. 2.4: Simulated log-enveloped beamformed PW imaging with 16 transmissions and different range of angles. Top row: $\pm 10, \pm 20, \pm 30$ and \pm 40 degrees from left. Bottom row: $\pm 50, \pm 60, \pm 70$ and \pm 80 degrees from left

Fig. 2.5: Simulated log-enveloped beamformed SAPW imaging with 16 transmissions and different range of angles. Top row: $\pm 10, \pm 20, \pm 30$ and \pm 40 degrees from left. Bottom row: \pm 50 , $\pm 60, \pm 70$ and \pm 80 degrees from left

Fig. 2.6: The effect of the range of transmission angles of PWs on the CNR of reconstructed anechoic cysts at different depths in PW (dashed lines) and SAPW (solid lines) imaging methods

Fig. 2.7: The effect of the range of transmission angles of PWs on the CNR of reconstructed anechoic cysts at different lateral locations of PW (dashed lines) and SAPW (solid lines) imaging methods

Fig. 2.8: Simulated log-enveloped beamformed PW without dynamic apodization (from left), PW with dynamic apodization in receiving, SAPW without dynamic apodization and SAPW with dynamic apodization in both transmitting and receiving imaging using the optimal configuration of PW in tissue mimicking phantom 30

Fig. 2.9: Line profile comparison between $P W$ and SAPW without and with dynamic apodization of Fig. 2.8 at different depth at the $0 \mathrm{~mm}$ lateral distance

Fig. 2.10: CNR comparison between PW and SAPW without and with dynamic apodization corresponding to Fig. 2.8 at different depths and at the $0 \mathrm{~mm}$ lateral distance

Fig. 2.11: The singular value distributions at three different frequencies with optimal configuration of PW imaging: central frequency, 5.0 MHz (left), 1.0 MHz (centre), and 7.0 MHz (right)

Fig. 2.12: Singular value distributions at three different frequencies with $P W$ transmission angles of \pm 60 degrees: central frequency of $5.0 \mathrm{MHz}$ (left), $1.0 \mathrm{MHz}$ (centre), and $7.0 \mathrm{MHz}$ (right) 
Fig. 2.13: Simulated log-enveloped beamformed PW (left) and SAPW (right) imaging using the optimal configuration of PW and $1 \mathrm{~dB}$ white Gaussian noise added to RF signal .............. 34

Fig. 2.14: Simulated log-enveloped beamformed PW (left) and SAPW (right) imaging in tissue mimicking phantom with $16 \mathrm{PW}$ transmissions, transmission angles of \pm 40 degrees and $1 \mathrm{~dB}$

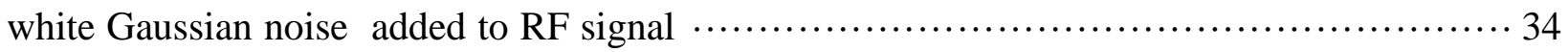

Fig. 2.15: Simulated log-enveloped beamformed PW imaging of point scatters with 16 transmissions and different range of angles. Top row: $\pm 10, \pm 20, \pm 30$ and \pm 40 degrees from left.

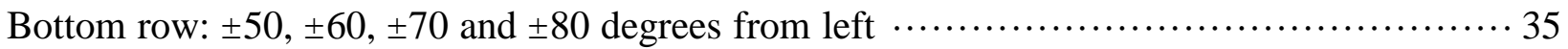

Fig. 2.16: Simulated log-enveloped beamformed SAPW imaging of point scatters with 16 transmissions and different range of angles. Top row: $\pm 10, \pm 20, \pm 30$ and \pm 40 degrees from left.

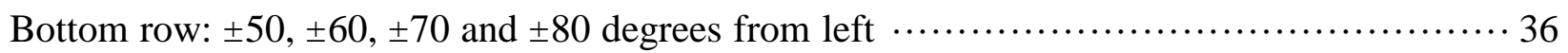

Fig. 2.17: The performance of the range of transmission angles with constant 16 transmission PWs and its effect on lateral resolution at different depths of PW (dashed lines) and SAPW (solid lines) imaging methods

Fig. 2.18: The performance of the range of transmission angles with 16 transmission PWs and its effect on the axial resolution at different depths of PW (dashed lines) and SAPW (solid lines)

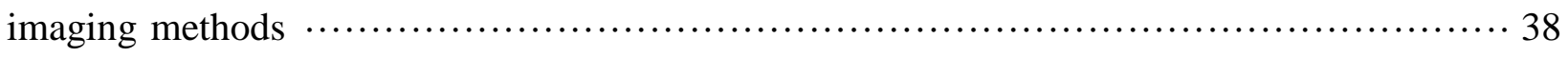

Fig. 2.19: The performance of the range of transmission angles with 16 transmission PWs and its effect on lateral resolution at different lateral distances of PW (dashed lines) and SAPW (solid lines) imaging methods

Fig. 2.20: The performance of the range of transmission angles with 16 transmission PWs and its effect on the axial resolution at different lateral distances of PW (dashed lines) and SAPW (solid lines) imaging methods

Fig. A.1: The experimental results of the tissue mimicking phantom in 16 transmission of PW with range of transmission of \pm 15.88 degrees. PW imaging (left,), SAPW imaging (middle) and conventional B-mode imaging (right) 
Fig. A.2: The experimental results of the tissue mimicking phantom in 16 transmission of PW with range of transmission of \pm 40 degrees. PW imaging (left,), SAPW imaging (middle) and conventional B-mode imaging (right)

Fig. B.1: Simulated log-enveloped beamformed imaging of anechoic soft tissue phantom with 16 transmissions with different sequences of the quarter of Hadamard encoded transmissions …53

Fig. B.2: Contrast-to-noise value anechoic inclusion of SAPW of different range of transmission angle from \pm 10 degrees to \pm 80 degrees and different quarter Hadamard transmissions at different

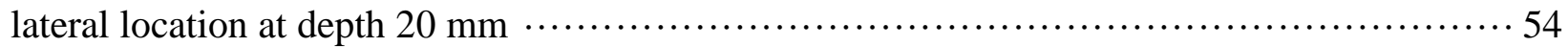

Fig. B.3: Contrast-to-noise value anechoic inclusion of SAPW of different range of transmission angle from \pm 10 degrees to \pm 80 degrees and different quarter Hadamard transmissions at different

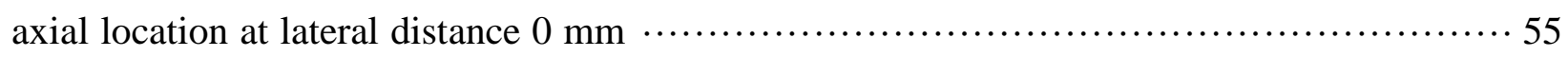

Fig. B.4: Simulated log-enveloped beamformed imaging of anechoic soft tissue phantom with combination of \pm 20 degrees $\mathrm{PW}$ transmissions and $4 \mathrm{r}$ quarter of Hadamard encoded transmissions 


\section{LIST OF ACRONYMS AND ABBREVIATIONS}

CNR Contrast-to-noise ratio

PW Plane wave

RF Radiofrequency

SAPW Synthetic-aperture plane wave

SNR Signal-to-noise ratio

STA Synthetic transmit aperture

SVD Singular value decomposition 


\section{Chapter 1}

\section{Introduction}

\subsection{Ultrasound Imaging}

Ultrasound imaging has been proven to be a safe, relatively simple, inexpensive, and realtime modality for imaging soft tissue. In medical ultrasound, the array of fixed number of elements is usually stationary and the lateral scan of a focused ultrasound beam is implemented electronically. The current conventional ultrasound imaging is called B-mode imaging. It is one of the most commonly used modes in which the radiofrequency (RF) signals are acquired after sending focused beams along one direction sequentially in time [1].
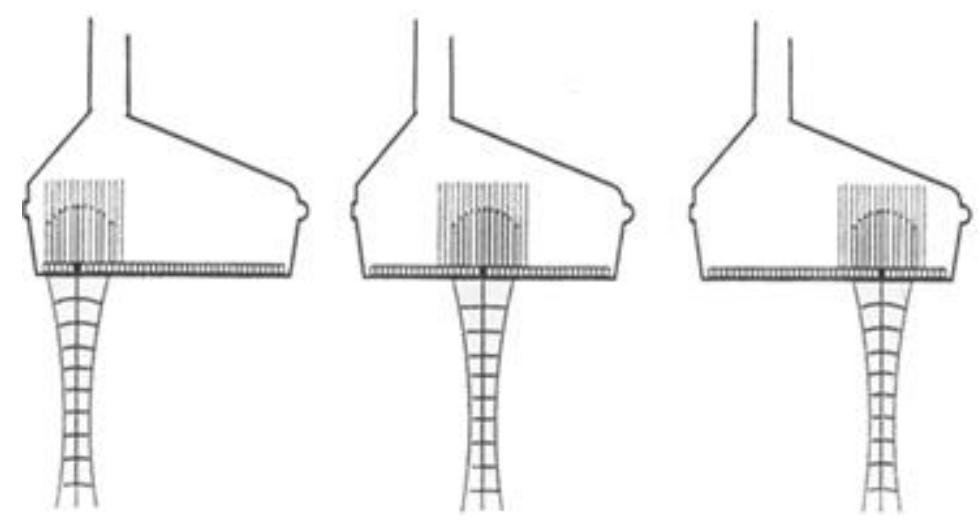

Fig 1.1. Basic principle of B-mode ultrasound imaging. 
The acquisition rate is limited by the maximum frame rate, $f_{B}$,

$$
f_{B}=\frac{c}{2 Z N}
$$

where the image has $N$ A-lines to a maximum depth of $Z$ and $c$ is the speed of sound. A disadvantage of the B-mode imaging is that the images are only optimally focused around the focal zone. Since the B-mode images are only focused at one depth due to the single transmission focus, spatial resolution and CNR of the B-mode ultrasound images still needs improvement at locations outside of the transmission focus. This can be overcome by making compound imaging using a multiple number of foci; however, this method would correspondingly decrease the frame rate [1].

Synthetic Transmit Aperture (STA) imaging [1] is a method to solve the disadvantages of B-mode imaging. A single element of the transducer transmits a semi-spherical wave into a large to-be-imaged region. In each transmission, all the elements receive the backscattered RF signals from the medium and a low resolution image is reconstructed using the delay-and-sum algorithm. All the low resolution images are combined to form a high-resolution image [2], [3].

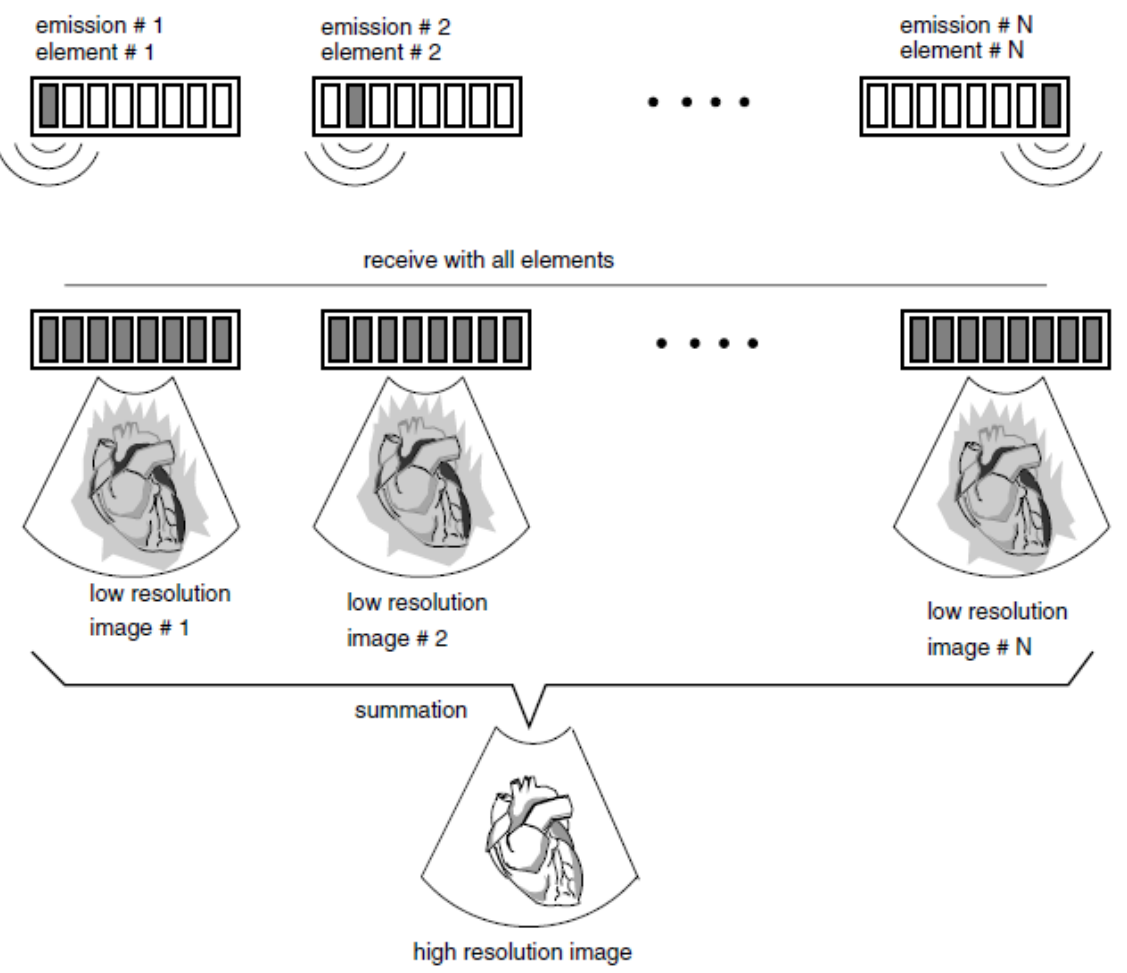

Fig. 1.2. Basic principle of synthetic aperture ultrasound imaging (from [1]). 
A low resolution image is only focused in receive due to the un-focused transmission. However, dynamic focusing in STA can be achieved at every point of the image to overcome the disadvantage of B-mode image. Focusing is performed by calculating the time it takes the ultrasound wave to travel from the transmitting element to the imaging point and then back to the receiving element [1].

$$
t\left(i_{t}, i_{r}\right)=\frac{\left|r_{p}-r_{t}\left(i_{t}\right)\right|+\left|r_{p}-r_{r}\left(i_{r}\right)\right|}{c}
$$

where $t\left(i_{t}, i_{r}\right)$ is the time to receive the proper signal, $r_{p}$ is the imaging point, $r_{t}\left(i_{t}\right)$ is the position of the transmitting element $i_{t}$, and $r_{r}\left(i_{r}\right)$ is the position of the receiving element $i_{r}$. This dynamic focusing can be performed for every imaging point. This focusing calculation produces transmit and receive focus for all points in the image; therefore, a high resolution image can be produced by compounding the low resolution images. However, STA has low SNR since only one or a small number of elements is selected for transmission.

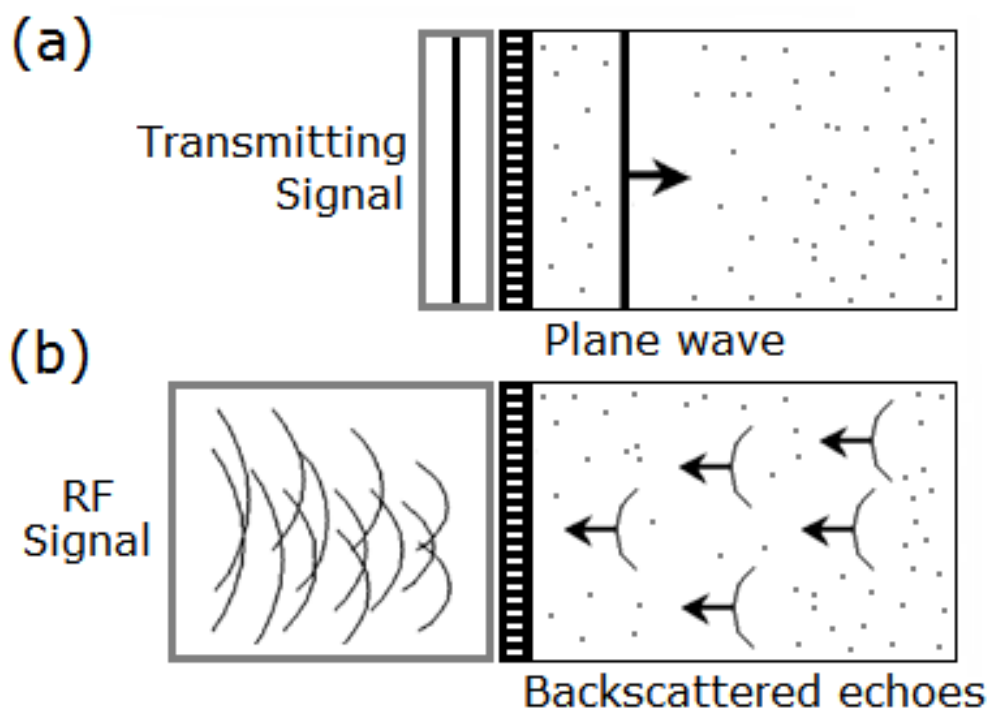

Fig. 1.3. Schematic representation of the single transmit PW method: (a) the ultrasonic array insonifies the medium using a PW transmission without steering, and (b) the backscattered RF signals are recorded by the transducer array. 
PW imaging (Fig. 1.3) can solve for the low SNR problem of STA because a whole aperture is used for each transmission. Currently, coherent PW compounding method proposed by Montaldo et al. [4] can reach the same image quality as the optimal multifocus B-mode imaging while preserving high frame rates. Idea of PW is not new although the capabilities of image enhancement were developed recently. In 1979, Delannoy et al. [5], [6] proposed use of parallel processing method to simultaneously produce entire image frame using a single pulse. This method enables 1000 frames per second (fps) with 70 lines per frame. In the early 1990s, Lu and Greenleaf proposed for achieving high frame rate by using non-diffracting beam [7]-[9]. Later Lu proposed the idea of a spatial compounding with different limited diffraction beams or steered PWs. Compounding incoherently and coherently would result in reducing speckle and enhancing resolution, respectively [10]-[12]. In late 1990s and early 2000s, Fink et al. improved the idea of PW insonification to achieve ultrafast frame rates (> $5000 \mathrm{fps}$ ) [13]-[15]. PW ultrasound imaging has been studied [16], [17] and developed in the field of medical ultrasound imaging.

Contrary to incoherent compound imaging, coherent wave compounding has not been extensively studied [4]. Incoherent compound imaging is mainly associated with an addition of the several image frames after envelope calculation (not in phase) in an attempt to cancel out random variations (noise) and hence increase the signal to noise ratio [17], [18]. Coherent compound imaging is addition of the several image frames before envelope calculation (in phase) in an attempt to enhance the spatial resolution. Coherent compounding PW shows strong conceptual analogies with the STA [19], [20]. It has been intensely studied in the past that STA could give better images than conventional B-mode images. A problem in STA is the poor SNR in the images. Coherent PW imaging solves at least partially these limitations of STA because it uses full aperture for a transmission of the PW. Moreover, a current method proposed by Montaldo et al. can reach the optimal multifocus B-mode image quality while preserving high frame rates. Optimal multifocus B-mode image is the image that all imaging regions are covered within the focal zone. To provide the optimal multifocus image, the transmitted focal depths must be separated by a maximal distance corresponding to depth of field $\Delta z=7 \lambda F^{2}$ [21] where $\lambda$ is the central wavelength of transmitted wave and $F$ is the F-number. 
(a)

Transducer

\section{IIIIIIIIIII}

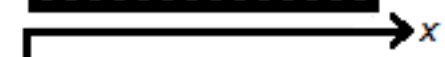

Fig. 1.4. (a) Axis convention, (b) distance to the point $(x, z)$ and back to transducer for a PW, and (c) for a PW of angle $\alpha$ steering.

For a PW, the travelling time to the point $(x, z)$ and back to transducer element located at $x_{1}$ depends on the inclination angle $\alpha$ of transmission and the speed of the sound in the medium, as shown in Fig. 1.4. The time required for the transmitted wave to reach the point $(x, z)$ is:

$$
t_{\text {transmit }}(\alpha, x, z)=\frac{z \cos \alpha+x \sin \alpha}{c}
$$

Since the wave front of the each PW is considered for calculation, the time required to receive a wave from point $(x, z)$ to the transducer element located at $x_{1}$ is:

$$
t_{\text {receive }}\left(x_{1}, x, z\right)=\frac{\sqrt{z^{2}+\left(x-x_{1}\right)^{2}}}{c}
$$

because each scatterer produces the spherical wave as the echoes. Therefore, the total time for a PW insonification is:

$$
t\left(\alpha, x_{1}, x, z\right)=t_{\text {transmit }}(\alpha, x, z)+t_{\text {receive }}\left(x_{1}, x, z\right)
$$

The image quality can be improved by compounding the images obtained with several PWs of different steering. When the PW is required to be given an inclination (or steering) $\alpha$, the 
proper delay is required to be applied to the transmitting element. The delay is expressed in (1.6) which depends on the steering angle of the PW and pitch of the transducer:

$$
d=\Delta n p \sin \alpha / c
$$

where $d$ is the delay that needs to be applied for the inclination $\alpha, p$ is the pitch which is the center-to-center distance between the neighboring elements, $\Delta n$ is the difference in the element numbers of the transducer from the reference transducer element, and $c$ is the speed of the wave travelling in the medium.

Similar to the synthetic aperture imaging, once a single PW is transmitted, a low resolution image can be reconstructed by applying the delay-and-sum method using the total time travelled by the transmitting-and-receiving ultrasound wave calculated by (1.5). Afterwards, all low-resolution images from each PW are combined using the coherent compounding method to produce a final high-resolution image.

Figure 1.5 and 1.6 were the images acquired experimentally with a 128-element array of 4.5 MHz, $60 \%$ bandwidth, pitch of $0.33 \mathrm{~mm}, \mathrm{~F}$ number of 1.75 in both the emission and reception [4]. The lateral scan is done at a $\lambda / 2$ step size. There is no apodization in emission and in reception for either the focused or the compound method. The coherent compounding method performs as well as the optimal multifocus B-mode image, but with a higher frame rate. Table 1.1 shows the summary of the principal comparison values for the example analyzed in [4]. 

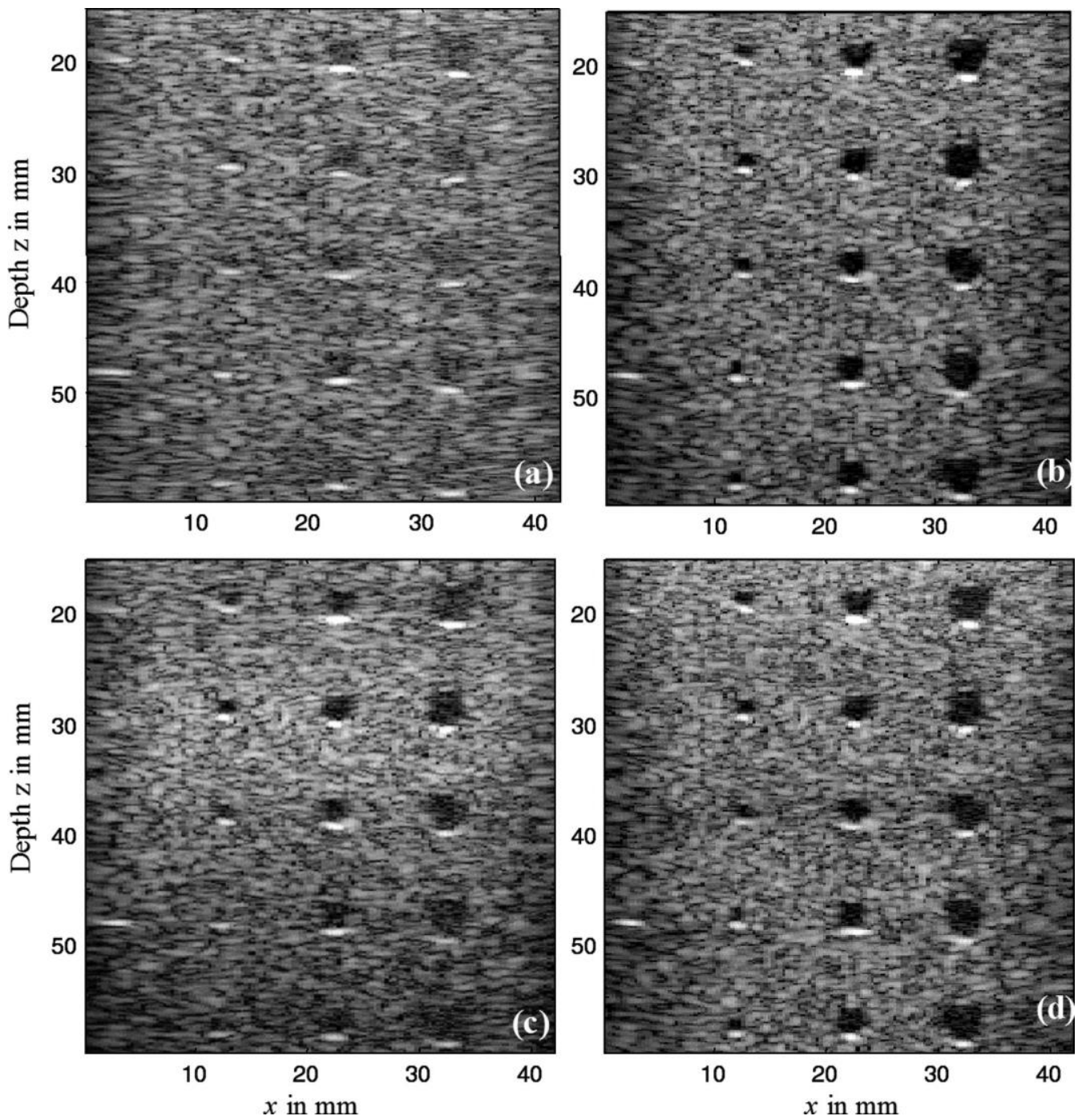

Fig. 1.5. (a) B-mode image obtained using a single PW transmission. (b) Coherent PW compounding using 71 angles with angular step size of $0.47^{\circ}$ (calculated using (2.13)). (c) Standard monofocal B-mode image using a single focal depth at $30 \mathrm{~mm}$. (d) Multifocal image using 4 successive focal depths. (from [4]) 

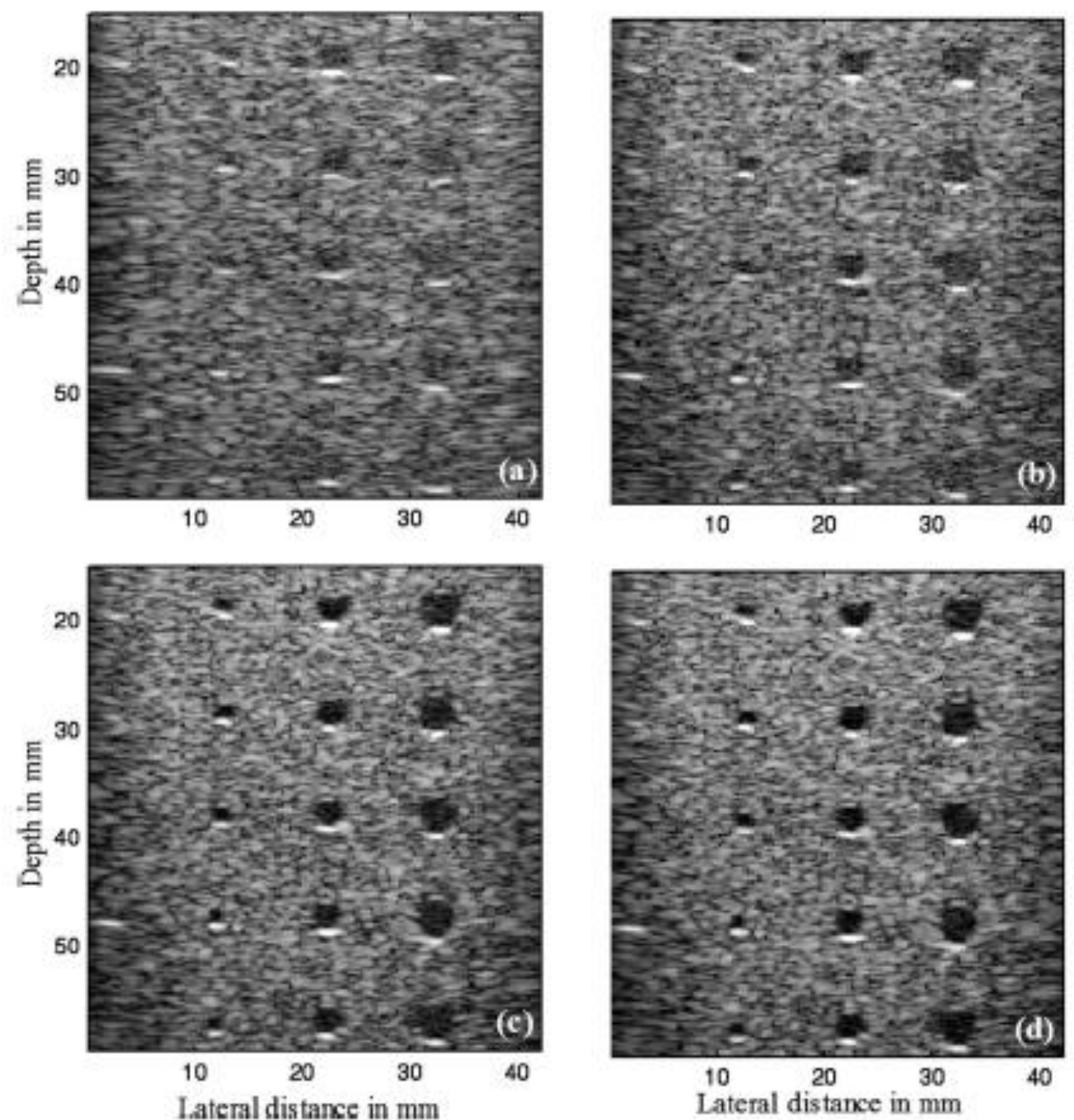

Fig. 1.6. Images of anechoic objects using different number of PW transmissions. (a) $1 \mathrm{PW}$, (b) 5 PWs separated $2^{\circ}\left(-4^{\circ},-2^{\circ}, 0^{\circ}, 2^{\circ}, 4^{\circ}\right)$, (c) 21 PWs separated $1^{\circ}$, and (d) 45 PWs separated $0.5^{\circ}$ (from [4]) 
Table 1.1. Quantitative comparison for the different methods. The SNR is relative to the single PW (from [4])

\begin{tabular}{lcccc}
\hline & $\begin{array}{c}\text { Lateral Resolution } \\
(\mathrm{mm})\end{array}$ & Frame Rate $(\mathrm{Hz})$ & Contrast $(\mathrm{dB})$ & SNR $(\mathrm{dB})$ \\
\hline B-mode (Monofocus) & 1.1 to 1.8 & 100 & 12 to 32 & 18 to 0 \\
B-mode (Multifocus 4) & 1.35 & 25 & 29 & 18 to 0 \\
B-mode (Multifocus & 1.1 & 8 & 33 & 18 to 0 \\
Optimal) & 1.8 & 12500 & 12 & 0 \\
PW (Single) & 1.1 & 1000 & 20 & 11 \\
PW Compound 12 & 1.1 & 270 & 30 & 16 \\
PW Compound 45 & 1.1 & 176 & 33 & 18 \\
PW Compound 71 & & & & \\
\hline
\end{tabular}

PW imaging still has lack of penetration due to lack of focusing in transmission [22]; however, the dynamic focusing in current image reconstruction techniques produces the transmit focus dynamic for all points in the image with the assumption that an infinite extent PW is transmitted. This assumption is not valid for the objects at large depth or not directly under the array. Consequently, the image quality for these locations will be compromised. The motivation of the research was to achieve more improvement with coherent PW imaging.

\subsection{Research Hypothesis and Specific Aims}

The hypothesis of this research is that the image reconstruction for PW imaging can be improved and optimized using a new proposed method called PW synthetic-aperture imaging reconstruction method. The specific aims of this research are to:

I. Generating simulated RF data in standard PW mode and new proposed synthetic aperture plane wave (SAPW) method using Field II ultrasound simulator.

II. Comparing the performances of standard PW and SAPW imaging method.

III. Optimizing and improving the PW imaging parameters - CNR and spatial resolutions - using SAPW. 


\subsection{Thesis Organization}

The remainder of this thesis is organized as following:

- Chapter 2 presents the manuscript: "Plane Wave Ultrasound Imaging Using Synthetic Aperture Image Reconstruction Techniques: A Simulation Study”, to be submitted to the journal of IEEE transactions on ultrasonics, ferroelectrics, and frequency control.

- Chapter 3 presents conclusion of the thesis and suggestion for future works.

- Appendix A presents a preliminary experimental performance validation of the proposed method.

- Appendix B presents the comparison of different Hadamard encoded transmissions using specifically selected and reduced transmissions. 


\section{REFERENCES FOR CHAPTER 1}

[1] J. A. Jensen, S. I. Nikolov, K. L. Lokke, and M. H. Pedersen, "Synthetic aperture ultrasound imaging", Ultrasonics, vol. 44, pp.e5-e15, 2006

[2] M. Karaman, A. Atalar, and H. Koymen, "VLSI circuits for adaptive digital beamforming in ultrasound imaging”, IEEE Trans. Med. Imag. vol.12, pp.711-720, 1993

[3] B.G. Tomov, J.A. Jensen, "Compact implementation of dynamic receive apodization in ultrasound scanners". in: Proceedings of the SPIE - Medical Imaging, pp. 260-271, 2004

[4] G. Montaldo, M. Tanter, J. Bercoff, N. Benech, and M. Fink, "Coherent planewave compounding for very high frame rate ultrasonography and transient elastography." IEEE Transactions on Ultrason. Ferroelectr. Freq. Control, vol. 56, pp. 489-506, 2009.

[5] B. Delannoy, R. Torgue, C. Bruneel, and E. Bridou, "Ultrafast electronicalimage reconstruction device" in Echocardiology, vol. 1, C. T. Lancee, Ed. (The Hague: Nijhoff, 1979), ch. 3, pp. 447-450.

[6] B. Delannoy, R. Torgue, C. Bruneel, E. Bridoux, J. M. Rouvaen, and H. LaSota, “Acoustical image reconstruction in parallel-processing analog electronic systems," J. Appl. Phys., vol. 50, pp. 3153-3159, May 1979.

[7] J.-Y. Lu and J. F. Greenleaf, "Pulse-echo imaging using a nondiffracting beam transducer," Ultrasound Med. Biol., vol. 17, no. 3, pp. 265-281, 1991.

[8] J.-Y. Lu and J. F. Greenleaf, "Ultrasonic nondiffracting transducer for medical imaging," IEEE Trans. Ultrason. Ferroelectr. Freq. Control, vol. 37, pp. 438-447, Sep. 1990.

[9] J.-Y. Lu and J. F. Greenleaf, "Experimental verification of nondiffracting X waves," IEEE Trans. Ultrason. Ferroelectr. Freq. Control, vol. 39, pp. 441-446, May 1992. 
[10] J.-Y. Lu, "2-D and 3-D high frame rate imaging with limited diffraction beams," IEEE Trans. Ultrason. Ferroelectr. Freq. Control, vol. 44, pp. 839-856, Jul. 1997.

[11] J.-Y. Lu, "Experimental study of high frame rate imaging with limited diffraction beams," IEEE Trans. Ultrason. Ferroelectr. Freq. Control, vol. 45, pp. 84-97, Jan. 1998.

[12] J. Cheng and J. Y. Lu, "Extended high frame rate imaging method with limited diffraction beams," IEEE Trans. Ultrason. Ferroelectr. Freq. Control, vol. 53, pp. 880-899, May 2006.

[13] L. Sandrin, S. Catheline, M. Tanter, X. Hennequin, and M. Fink, "Time resolved pulsed elastography with ultrafast ultrasonic imaging," Ultrason. Imaging, vol. 21, pp. 259-272, Oct. 1999.

[14] L. Sandrin, S. Catheline, M. Tanter, C. Vinçonneau, and M. Fink, "2D transient elastography," Acoust. Imaging, vol. 25, pp. 485-492, Jan. 2000.

[15] L. Sandrin, M. Tanter, S. Catheline, and M. Fink, "Shear modulus imaging using 2D transient elastography," IEEE Trans. Ultrason. Ferroelectr. Freq. Control, vol. 49, no. 4, pp. 426-435, Apr. 2002.

[16] S. K. Jespersen, J. E. Wilhjelm, and H. Sillesen, "Multi-angle compound imaging," Ultrason. Imaging, vol. 20, pp. 81-102, Apr. 1998.

[17] R . R. Entrekin, B. A. Porter, H. H. Sillesen, A. D. Wong, P. L. Cooperberg, and C. H. Fix, "Real-time spatial compound imaging: Application to breast, vascular, and musculoskeletal ultrasound," Semin. Ultrasound CT MR, vol. 22, pp. 50-64, Feb. 2001.

[18] F. Forsberg, "Ultrasonic biomedical technology; marketing versus clinical reality," Ultrasonics, vol. 42, pp. 17-27, Apr. 2004. 
[19] J. Jensen, O. Holm, L. J. Jerisen, H. Bendsen, S. I. Nikolov, B. G. Tomov, P. Munk, M. Hansen, K. Salomonsen, J. Hansen, K. Gormsen, H. M. Pedersen, and K. L. Gammelmark, "Ultrasound research scanner for real-time synthetic aperture data acquisition," IEEE Trans. Ultrason. Ferroelectr. Freq. Control, vol. 52, no. 5, pp. 881-891, May 2005.

[20] T. Misaridis and J. A. Jensen, "Use of modulated excitation signals in medical ultrasound. Part III: High frame rate imaging," IEEE Trans. Ultrason. Ferroelectr. Freq. Control, vol. 52, no. 2, pp. 208-219, Feb. 2005.

[21] G. S. Kino, Acoustic Waves, Devices Imaging and Analog Signal Processing. Upper Saddle River, NJ: Prentice Hall, 1987, p. 191.

[22] P. Song, M. W. Urban, A. Manduca, J. F. Greenleaf, and S. Chen, "Coded Excitation Plane Wave Imaging for Shear Wave Motion Detection", IEEE Trans. Ultrason. Ferroelect. Freq. Contr., vol. 62, no. 7, pp. 1356-1372, Jul. 2015 


\section{Chapter 2}

\section{Plane Wave Ultrasound Imaging Using Synthetic Aperture Image Reconstruction Techniques: A Simulation Study}

This chapter presents a manuscript which has been submitted to the journal of IEEE Transactions on Medical Imaging on January 25, 2016.

Manuscript number: TMI-2016-0065 


\title{
Plane Wave Ultrasound Imaging Using Synthetic Aperture Image Reconstruction Techniques: A Simulation Study
}

\author{
Dae-Myoung Yang, Ping Gong, Jahan Tavakkoli, Yuan Xu* \\ Department of Physics, Ryerson University, Toronto, Ontario, Canada
}

*Corresponding author

Abstract - Ultrasound imaging based on transmitting plane waves (PW) enables ultrafast imaging. Coherent compounding of steered PW in multiple directions can result in high spatial resolution. PW ultrasound compounding imaging can reach the image quality of optimal multifocus image in terms of contrast, SNR and lateral resolution with a smaller number of PW transmissions while preserving high frame rate. In the PW image reconstruction, it was assumed that an infinite extent $P W$ is emitted from an array probe to the target region. However, because of the finite size of the ultrasound array, this assumption is not valid for points that are located at the edge or outside of the ultrasound beam. In this study, we propose a new image reconstruction algorithm designed for PW imaging without using the intrinsic PW assumption. In this paper, we propose a syntheticaperture PW (SAPW) imaging method, in which the equivalent RF data in synthetic aperture imaging are estimated from the PW RF signals to improve the image quality in terms of depth of penetration and field of view using contrast-to-noise ratio (CNR) as a quantitative metric. PW imaging can be treated as a special case of delay-encoded transmission. The measured RF data in PW imaging was first decoded in the frequency domain using a pseudoinverse algorithm to estimate the RF data which is equivalent to conventional synthetic transmit aperture (STA) RF signal when a single-element transmission is used. Equivalent STA RF data were then used to reconstruct ultrasound 
images through the standard STA method. In our paper, the PW RF signals were generated in numerical simulations using Field II. The SAPW imaging was compared with the PW imaging in the numerical simulations. The CNR value of the images of a simulated anechoic soft tissue cyst phantom with a scattering and attenuated background medium (attenuation coefficient $=0.6 \mathrm{~dB} /(\mathrm{cm} \cdot \mathrm{MHz})$ ) was improved. Also, applying dynamic apodization improves depth of the penetration due to the signal amplitude increase at large depth. The axial and lateral spatial resolutions of images of a point scatter phantom in SAPW are similar to PW imaging. It was observed that the main improvement in image quality of the SAPW in comparison to the PW is an increase in the imaging depth of penetration and the field of view.

Index Terms - Plane Wave Imaging, Encoded Transmission, Singular Value Decomposition, Pseudoinverse, Synthetic Aperture Imaging, Field of View, Depth of Penetration, Contrast-to-Noise Ratio, Spatial Resolution.

\section{INTRODUCTION}

Ultrasound imaging has been developed over the past 50 years. It is safe, inexpensive, portable, and real-time [1]. The concept of plane wave (PW) ultrasound imaging led to ultrafast frame rates higher than $5000 \mathrm{fps}$ [2]-[4]. The ultrasound images obtained from this ultrafast mode suffers from low image quality in terms of spatial resolution and contrast. In 2009, Montaldo et al. [5] proposed the coherent compounding PW imaging method to improve spatial resolution and contrast, comparable to those of the optimal multifocus B-mode imaging technique, where all imaging regions are focused.

For coherent PW imaging, an array of elements is used as a transmitter and then the same array can be used as receivers. The backscatter signals are received by all elements in the transducer when a single PW covers the region of interest. Multiple PWs at different angles are transmitted and then received. Images that are produced by each RF echo are coherently 
compounded. Coherent compounding PW imaging has strong conceptual analogies to the synthetic aperture method [6], [7]. In synthetic aperture method, each element in an ultrasound transducer array is excited consecutively and the complete set of RF echoes for all the transmitand-receive combinations are recorded. The set of received RF echoes corresponding to the same transmitter would be processed to reconstruct a low-resolution image using the delay-and-sum beamforming [8]. Afterwards, all low resolution images can be coherently compounded to produce a high resolution image. However, the traditional synthetic aperture imaging has fundamentally low signal-to-noise ratio in the RF signal because a single element is used in each transmission. Coherently compounding PW imaging is one of the approaches to solve this low signal-to-noise limitation by generating a much stronger wave field than synthetic aperture imaging.

In PW image reconstruction, it was assumed that an infinite extent PW is emitted from the array probe to the target region. However, because of the finite size of the ultrasound array, this assumption is not valid for points that are located at the edge or outside of the ultrasound beam. In this paper, we propose a new image reconstruction algorithm called synthetic-aperture plane wave (SAPW) imaging designed for PW imaging without using this assumption. In SAPW, the equivalent RF data in synthetic aperture imaging are estimated from the PW RF signals in the frequency domain using a pseudoinverse algorithm. [9]-[11]

The paper is organized as follows: In Section II, a theoretical method of SAPW and its simulation parameter is introduced. In Section III, a quantitative simulation comparison of both PW and SAPW approaches is presented with a detailed comparison of field of view and depth of penetration using the quantitative value of contrast-to-noise ratio (CNR) as a quantitative metric. In Section IV, the conclusion of this paper is presented. 


\section{THEORY}

A. Synthetic Aperture Plane Wave Imaging: Estimating RF Data Generated by Individual Array Elements

The realistically generated ultrasonic PW is a wave front that is generated with multiple elements in the transducer; therefore, it is not infinitely extended. This wave front is generated by interference of individual spherical waves produced by each array element. For coherent PW compounding, there would be multiple PWs transmitting in RF data acquisition to form one high-resolution image. For each transmission with certain inclination angle $\alpha$, a discrete amount delay $d$, is applied on each transducer element depending mainly on the transmitting element number and the inclination angle.

Each delay $d_{w n}$ is applied for each element $n$ (between 1 and $N$ ) and each transmission order $w$ (between 1 and $W$ ). $d_{w n}$ corresponds to a particular transmission and element position. Each row of matrix $\mathbf{D}$ includes delays applied to all transmission elements in one transmission event, and each column of matrix $\mathbf{D}$ includes delays applied to each element position. Therefore, the delay matrix, $\mathbf{D}$ is defined by a $W$-by- $N$ matrix.

$$
\mathbf{D}=\left[\begin{array}{ccc}
d_{11} & \cdots & d_{1 N} \\
\vdots & \ddots & \vdots \\
d_{W 1} & \cdots & d_{W N}
\end{array}\right]
$$

The encoded matrix $\mathbf{A}$ is required to estimate STA equivalent RF data using PW transmission RF data. According to the time-shift property of the Fourier transform, the delay $d_{w n}$ can be express in frequency domain. The delay encoded term, $A_{w n}(f)$, and the matrix $\mathbf{A}$ are defined as:

$$
\begin{gathered}
A_{w n}(f)=e^{-i 2 \pi f d_{w n}} \\
\mathbf{A}=\left[\begin{array}{ccc}
e^{-i 2 \pi f d_{11}} & \cdots & e^{-i 2 \pi f d_{1 N}} \\
\vdots & \ddots & \vdots \\
e^{-i 2 \pi f d_{W 1}} & \cdots & e^{-i 2 \pi f d_{W N}}
\end{array}\right]
\end{gathered}
$$

where $f$ is a given frequency in the spectrum, and $\mathbf{A}$ is a matrix with elements of $A_{w n}(f)$.

Each $w$ transmission of PW RF data, $m_{w m}(t)$, is acquired by RF data that has been transmitted with a spherical wave from each element, $p_{n m}(t)$, with discrete delay to produce PW 
front that has $\alpha$ inclination. $p_{n m}(t)$ is STA equivalent RF data In the frequency domain by transforming all RF data in time using Fourier transform, this yields

$$
\begin{gathered}
A_{w n}(f) P_{n m}(f)=M_{w m}(f) \\
\mathbf{A P}=\mathbf{M}
\end{gathered}
$$

where $P_{n m}(f)$ is the Fourier transform of signal $p_{n m}(t), M_{w m}(f)$ is the Fourier transform of signal $m_{w m}(t) . p_{n m}(t)$, STA equivalent RF data, can be estimated using a linear dependent combination in frequency domain by calculating $P_{n m}(f)$ and applying inverse Fourier transform to it.

$$
\left[\begin{array}{ccc}
e^{-i 2 \pi f d_{11}} & \cdots & e^{-i 2 \pi f d_{1 N}} \\
\vdots & \ddots & \vdots \\
e^{-i 2 \pi f d_{W 1}} & \cdots & e^{-i 2 \pi f d_{W N}}
\end{array}\right]\left[\begin{array}{ccc}
P_{11} & \cdots & P_{1 M} \\
\vdots & \ddots & \vdots \\
P_{N 1} & \cdots & P_{N M}
\end{array}\right]=\left[\begin{array}{ccc}
M_{11} & \cdots & M_{1 M} \\
\vdots & \ddots & \vdots \\
M_{W 1} & \cdots & M_{W M}
\end{array}\right]
$$

From (5) and its expanded version (6), the matrix $\mathbf{P}$ can be solved since spectrum of PW data, matrix $\mathbf{M}$, and encoding matrix, $\mathbf{A}$, are known. The matrix $\mathbf{P}$ is the data spectrum of the equivalent STA data. Using matrix $\mathbf{P}, \mathrm{RF}$ data of synthetic aperture imaging can be estimated by an inverse Fourier transform of $\mathbf{P}$, and a high-resolution image can be achieved by compounding low-resolution images reconstructed from $p_{n m}(t) \mathrm{RF}$ data corresponding to each transmitter.

\section{B. Singular Value Filter (SVF): Optimizing STA Equivalent RF Data Obtained from Pseudoinverse Using Singular Values}

For solving for $\mathbf{P}$ in (5), the pseudo-inversion is needed for matrix $\mathbf{A}$ since it is not a square matrix, in general. Matrix $\mathbf{P}$ is derived using:

$$
\mathbf{P}=\mathbf{A}^{+} \mathbf{M}
$$

where $\mathbf{A}^{+}$is the pseudo-inversed matrix of $\mathbf{A}$. 
Pseudoinversion is the application of mathematical operation using singular value decomposition (SVD) [12]. SVD decomposes the $w$-by- $n$ matrix $\mathbf{A}$ into the two orthogonal matrixes and a diagonal matrix that consists of values that are called the singular values of $\mathbf{A}$.

$$
\begin{gathered}
\mathbf{A}=\mathbf{U} \boldsymbol{\Sigma} \mathbf{V}^{\mathrm{T}} \\
\mathbf{A}^{+}=\mathbf{V} \boldsymbol{\Sigma}_{\mathbf{1}}^{\mathbf{T}} \mathbf{U}^{\mathbf{T}}
\end{gathered}
$$

where $\mathbf{U}$ and $\mathbf{V}$ are unitary matrices, $\boldsymbol{\Sigma}$ is a diagonal matrix whose elements are the singular values of the original matrix $\mathbf{A} . \boldsymbol{\Sigma}_{\mathbf{1}}$ is the inverse of the diagonal part of matrix $\boldsymbol{\Sigma}$. If matrix $\mathbf{A}$ is not square or if it is square but not invertible, then it cannot be inverted. However, in the case of a non-square matrix $\mathbf{A}$, one can apply pseudoinversion because matrix $\boldsymbol{\Sigma}$ is always invertible. The pseudoinverse is a more general method that can be applied to all matrices. Matrix $\boldsymbol{\Sigma}$ is a diagonal matrix of $\sigma_{k}$ with as many singular values as the rank of matrix $\mathbf{A}$ arranged in order of descending value.

$$
\sigma_{1}>\sigma_{2}>\sigma_{3}>\cdots>\sigma_{m}>0 \quad(k=1: m, \operatorname{rank} \text { of } \mathbf{A})
$$

Since high $k$ numbers of singular values are smaller numbers, the undesirable noise that may exist in matrix $\mathbf{P}$ will be amplified during the pseudoinversion because of the inversion in constructing matrix $\Sigma_{1}$.

In order to optimize the matrix $\mathbf{P}$ to avoid amplified noise due to the inversing, a filter can be applied by cutting off the small singular values. Any singular value below a set cut-off value would not contribute to calculating matrix $\mathbf{P}$. The criteria chosen for the cut-off value was set by observing the singular value distribution of each frequency because the distributions of the singular values change at different frequencies. There are two a priori criteria that were used in this study. Ultrasound RF data is the most stable at the central frequency. All singular value components of the central frequency must be included because transmission at the central frequency gives the most stable RF data. As the frequency gets further away from the central frequency, the distribution of the singular value changes and contains a low number of singular values. Another criterion is to avoid amplification due to the inversion. Any singular value that is smaller than 1 would cause the amplification when it is inversed. Therefore, the cut-off value 
should be set below the lowest singular value at the central frequency and greater than or equal to 1 . Using these characteristics, the SAPW imaging can be stabilized.

$$
\sigma_{m, f_{o}}>\sigma_{\text {cut }- \text { off }} \geq 1
$$

Once the SAPW data or equivalent STA data are estimated, the image of SAPW is obtained using the standard delay-and-sum reconstruction in synthetic aperture imaging.

\section{Generating the Simulated RF Data with Field II Simulation Software}

Field II simulation software [13], [14] was used to simulate the PW RF signal in two dimensions. To generate the PW signals, each different PWs in different directions were transmitted using a linear phased array transducer with 64 elements. The angle step size was kept constant for all transmissions depending on the range of the angles. Linear phased array probe with 64 elements that has a half-wavelength pitch and a quarter-wavelength element width was excited with a one-cycle $5 \mathrm{MHz}$ sinusoidal pulse. The received signals were sampled at $20 \mathrm{MHz}$. The speed of sound was kept at $1540 \mathrm{~m} / \mathrm{s}$ and an attenuation of $0.6 \mathrm{~dB} /(\mathrm{cm} \cdot \mathrm{MHz})$ was assumed in the simulations to mimic the tissue phantom. The phantom was $40 \mathrm{~mm} \times 80 \mathrm{~mm} \times 10 \mathrm{~mm}$ (lateral $\times$ axial $\times$ elevation) with a scattering density of 10 scatters $/ \mathrm{mm}^{3}$. These background scatters are mathematical point scatters. The spatial distribution of the scatters was randomly distributed and their scattering strength followed a zero-mean Gaussian distribution. For measuring the depth of penetration and the field of view, contrast-to-noise ratio was used as a quantitative metric, and the phantom for this study included $5 \mathrm{~mm}$ diameter anechoic inclusions. For measuring the lateral and axial resolutions, the full width half maximum (FWHM) of the imaging value of the point scatters in a liquid medium with the same attenuation was used. These point scatters have 20 times the strength of the background scattering. 
Table 2.1. PW simulation parameters

\begin{tabular}{ll}
\hline Simulation Parameters & Default Values \\
\hline Central Frequency $\left(\mathrm{f}_{\mathrm{o}}\right)$ & $5 \mathrm{MHz}$ \\
Sampling Frequency $\left(\mathrm{f}_{\mathrm{s}}\right)$ & $20 \mathrm{MHz}$ \\
Number of Transmissions & 16 \\
Angular range of Transmissions & $10-80$ degrees \\
Cut-off Parameter for SVF & 1 \\
Phantom Size & $40 \times 80 \times 10 \mathrm{~mm}^{3}$ \\
Phantom Attenuation Coefficient & $0.6 \mathrm{~dB} /(\mathrm{cm} \cdot \mathrm{MHz})$ \\
Scattering Density & $10 \mathrm{scatters} / \mathrm{mm}^{3}$ \\
Speed of sound $\left(\mathrm{c}_{\mathrm{o}}\right)$ & $1540 \mathrm{~m} / \mathrm{s}$ \\
Number of Elements & 64 \\
Wavelength $\left(\lambda_{\mathrm{o}}\right)$ & $0.308 \mathrm{~mm}$ \\
Array Element Pitch & $\lambda / 2$ \\
Array Element Width & $\lambda / 4$ \\
Dynamic Range of the Images & $60 \mathrm{~dB}$ \\
\hline
\end{tabular}

The CNR of the anechoic inclusion was measured for quantitative metric. Referring to Figure 2.1, the CNR of an ultrasound image is measured using the following expression (12) [15]:

$$
C N R=\frac{\left|\mu_{i}-\mu_{o}\right|}{\sqrt{\sigma_{i}^{2}+\sigma_{o}^{2}}}
$$

where $\mu_{i}$ and $\mu_{o}$ are the mean envelop-detected pixel intensities in regions inside and outside the anechoic cyst, respectively, and $\sigma_{i}$ and $\sigma_{o}$ is the standard deviations inside and outside the cyst respectively. The total areas of the inside and outside measurement regions are equal for measuring the CNR. 


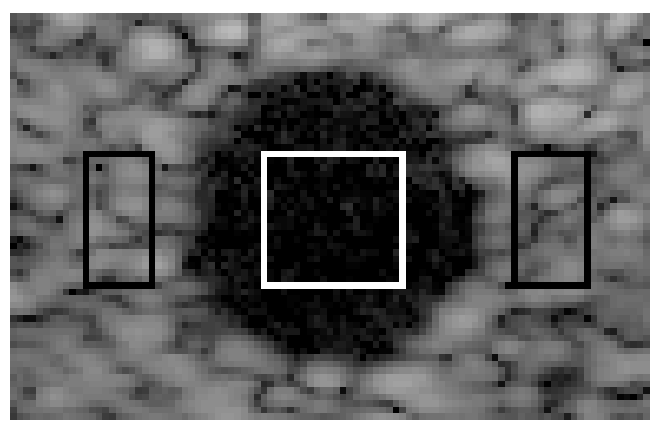

Fig. 2.1. Region of interest (ROI) in anechoic cyst simulation to estimate CNR. The white box indicates the ROI inside the cyst and black boxes indicates ROI outside the cyst. The mean and standard deviation of pixel values inside and outside the cyst were used to compute CNR as expressed in (12).

\section{RESULTS AND DISCUSSIONS}

\section{A. Depth of Penetration and Field of View Using Contrast-to-Noise Ratio (CNR) as a}

\section{Quantitative Metric}

There is an optimal configuration of PW imaging to obtain the same focusing quality as the optimal multifocus [5]. The step-size for angles of transmission and the number of transmission are determined by the total length of the aperture, wavelength at central frequency, and F-number as the following equations:

$$
\begin{gathered}
\alpha_{s}=\sin ^{-1}\left(\frac{\lambda_{o}}{L}\right) \\
w=\frac{L}{\lambda_{o} F}
\end{gathered}
$$

where $\alpha_{s}$ is the step-size of angles of transmission, $L$ is the total length of the aperture, and $F$ is the F-number. Previous studies have kept an F-number of 1.75 [5]. With the same F-number and the above parameter of the transducer, it is enough to use 19 transmissions with the step-size of 1.80 degrees insonifications of PWs to obtain the same focusing quality as the optimal 
multifocus from (13) and (14). Figure 2.2 shows the images reconstructed in both PW and SAPW, and Figure 2.3 shows the CNR comparison between the two methods.
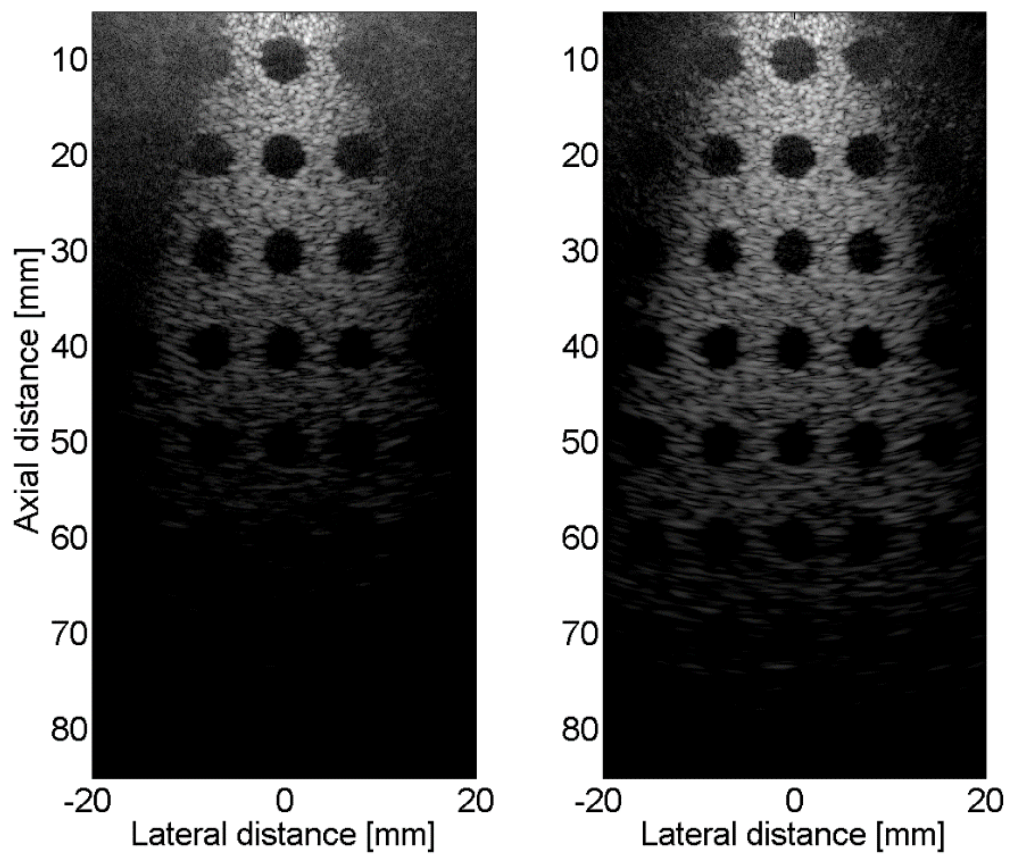

Fig. 2.2. Simulated log-enveloped beamformed PW (left) and SAPW (right) imaging using the optimal configuration of PW in tissue mimicking phantom.
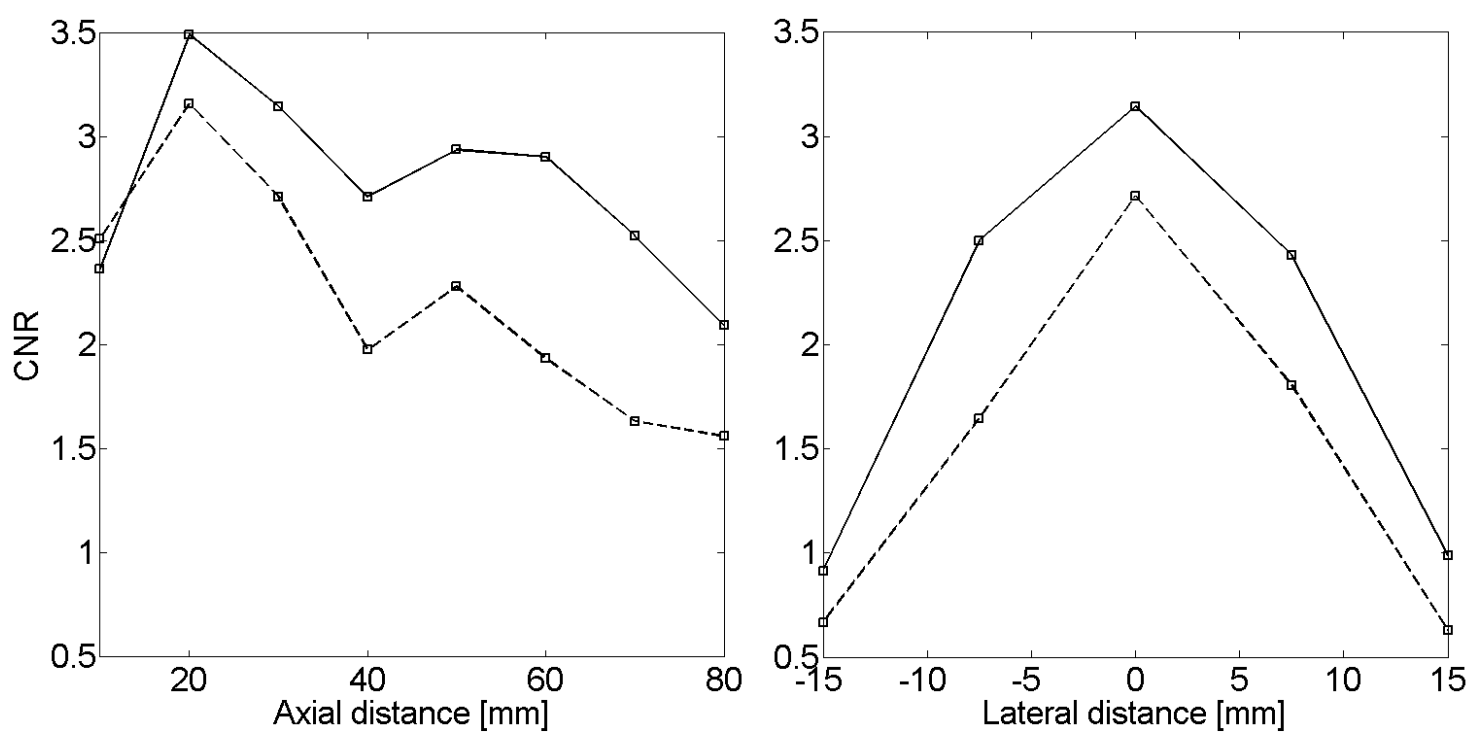

Fig. 2.3. CNR comparison between PW (dashed lines) and SAPW (solid lines) of Fig. 2.2 at different depth at the $0 \mathrm{~mm}$ lateral distance (left) and different lateral distance at depth $30 \mathrm{~mm}$ (right). 
There is no significant improvement with visual inspection of Figure 2.2 and CNR did not significantly improved in the imaging region under the aperture $(-5.0$ to $5.0 \mathrm{~mm})$ at the depth of penetration $(10,20,30 \mathrm{~mm})$. However, Figure 2.3 shows an increase in the depth of penetration and a slight improvement of the field of view. At $40 \mathrm{~mm}$ under the aperture, the CNR value of SAPW imaging was increased by $38.5 \%$ comparing to PW imaging. Also the CNR value of two anechoic inclusions at a lateral distance of $-7.5 \mathrm{~mm}$ and $7.5 \mathrm{~mm}$ show significant improvements as well, at $52.2 \%$ and $35.8 \%$ respectively. The number of the transmission and the step-size of angles of transmissions were changed because there is no significant improvement with the optimal configuration of PW imaging and the transmission of SAPW imaging, which is what SAPW is calculating does not vary with the F-number. For this simulation, a total of 16 transmissions of $\mathrm{PW}$ were used. The range of the transmission angles is from \pm 10 degrees to \pm 80 degrees, and they have the same number of transmissions, which means each range of the transmission angles has a different step-size. The 16 transmissions were chosen to have less transmission than the optimal configuration, and also to use a quarter transmission of conventional synthetic aperture imaging.

Figures 2.4 and 2.5 show the simulated PW and SAPW images with eight different ranges of the transmission angles, respectively. With visual inspection, the depth of penetration and field of view of the SAPW imaging show improvements. As the range of the angle increases, more improvements are observed. There is no apodization applied to any of these images. Since there is an attenuation of $0.6 \mathrm{~dB} /(\mathrm{cm} \cdot \mathrm{MHz})$, the depth of penetration is limited; however, SAPW imaging shows an improved depth of penetration over PW imaging. 

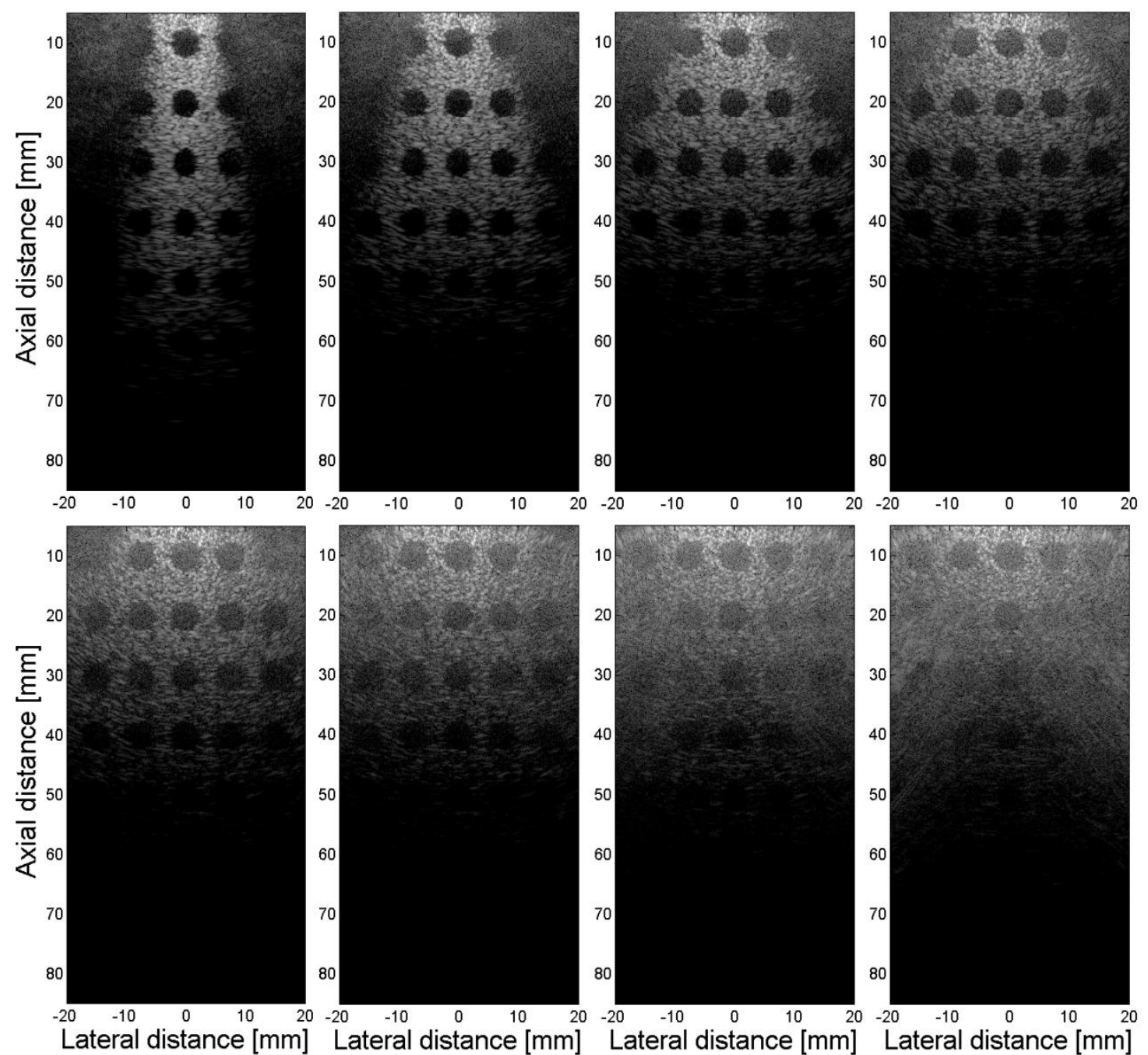

Fig. 2.4. Simulated log-enveloped beamformed PW imaging with 16 transmissions and different range of angles. Top row: $\pm 10, \pm 20, \pm 30$ and \pm 40 degrees from left. Bottom row: $\pm 50, \pm 60, \pm 70$ and \pm 80 degrees from left. 

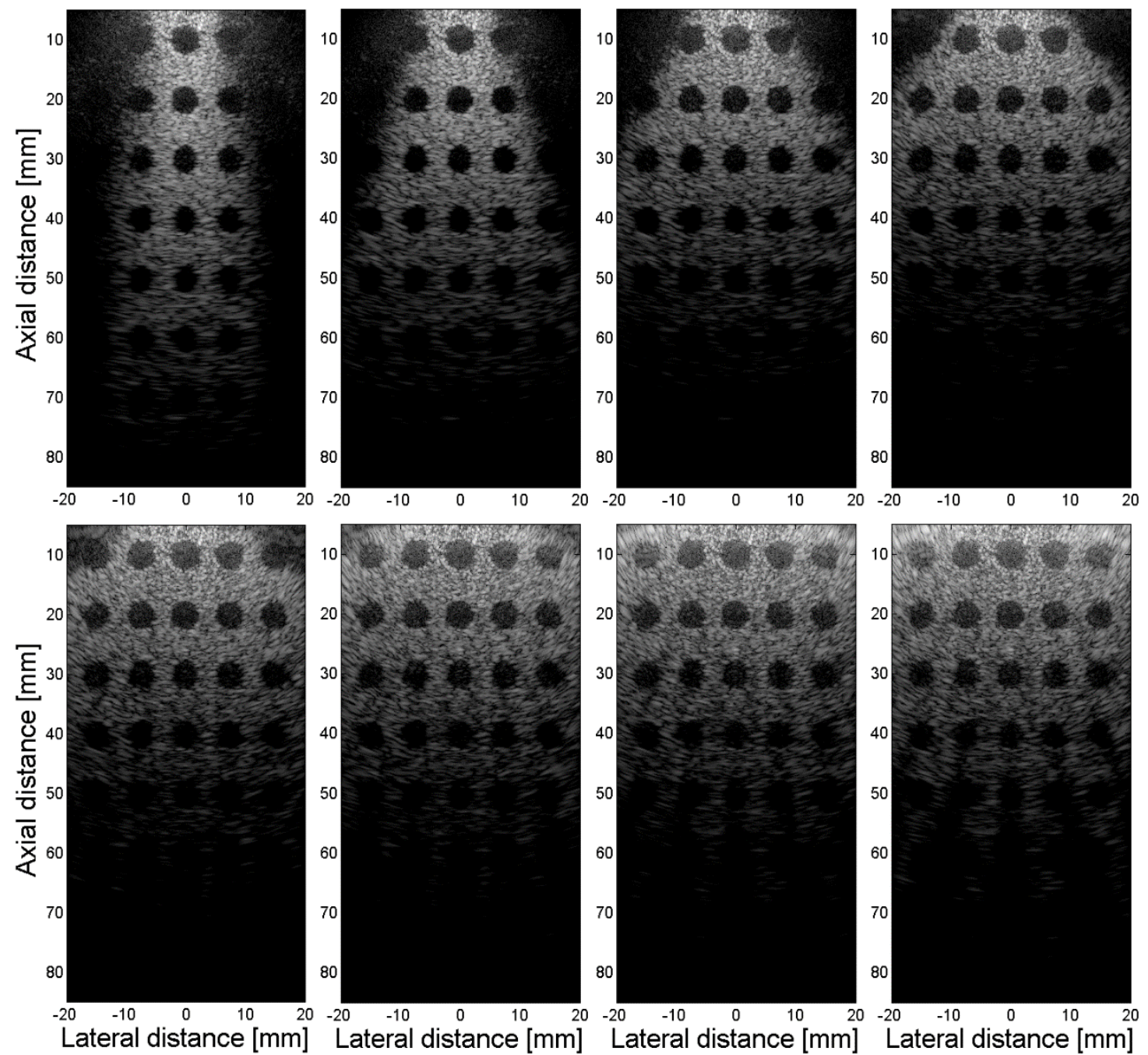

Fig. 2.5. Simulated log-enveloped beamformed SAPW imaging with 16 transmissions and different range of angles. Top row: $\pm 10, \pm 20, \pm 30$ and \pm 40 degrees from left. Bottom row: \pm 50 , $\pm 60, \pm 70$ and \pm 80 degrees from left.

Figure 2.6 and 2.7 show CNR vs. the range of transmission angles at different locations. PW imaging is proven to achieve superior image quality at the regions under the aperture; therefore, $\mathrm{CNR}$ of $\mathrm{PW}$ is better than SAPW in the range of angles within \pm 10 degrees where the transmission wave is concentrated in the shallow depth and region under the aperture. However, as the range of angles increases, the step-size of the angles increases as well because number of transmissions was kept constant throughout the study; therefore, CNR is decreasing as the range of transmission angle increases for anechoic cyst inclusions located at the centre of lateral 
distance. The cyst inclusions located in regions outside the aperture have the optimal CNR depending on the range of the transmission angle due to locations and transmission beam being scanned. Generally, the effect of SAPW shows greater improvement at deeper depth and at regions outside the aperture. Moreover, SAPW imaging would have better CNR values than PW imaging. This CNR decrease in PW imaging is due to limited number of transmissions, which can be compensated for by using the proposed SAPW image reconstruction method. The effect of the range of transmission angles of PWs on the CNR are shown in Figures 2.6 and 2.7.
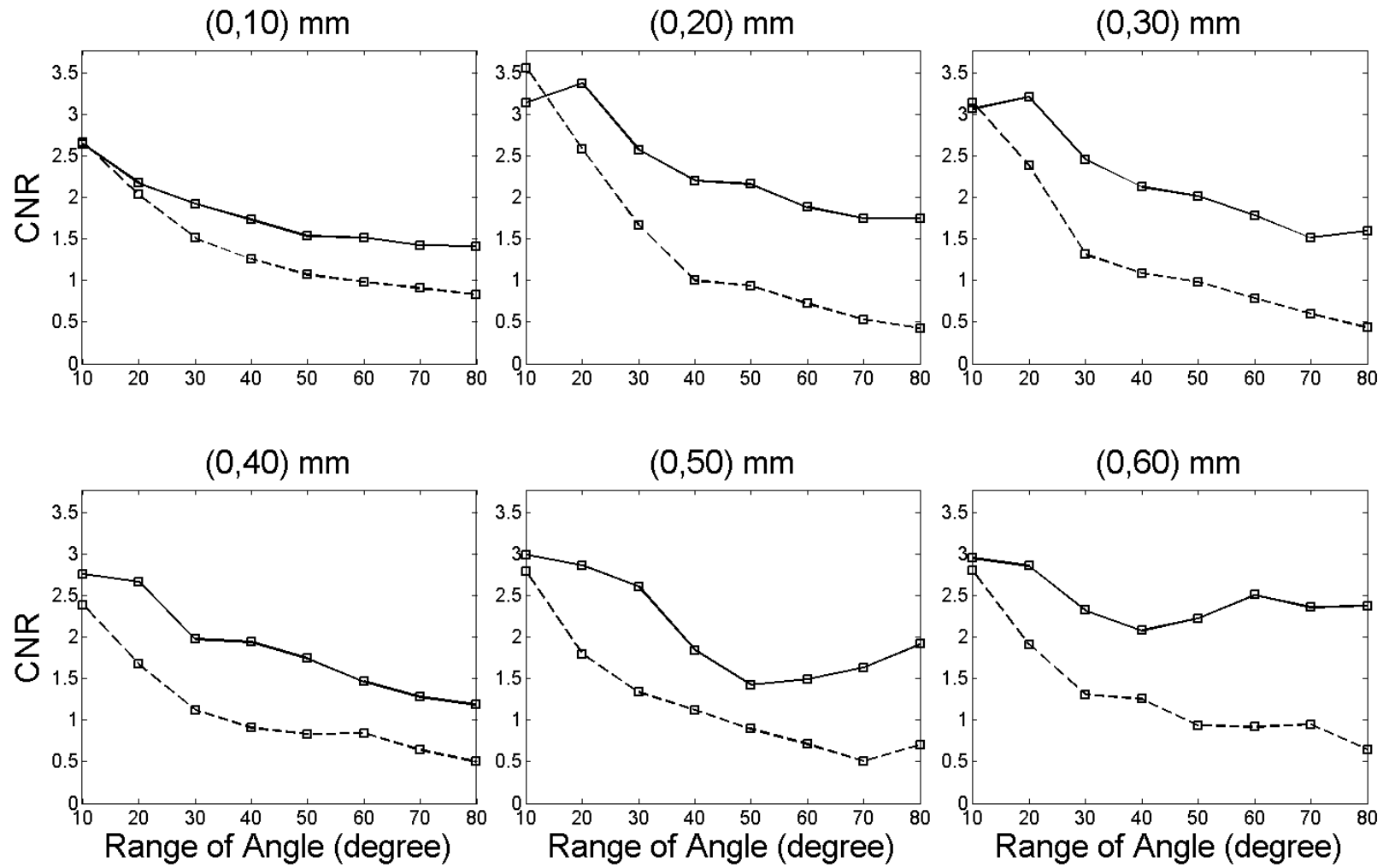

Fig. 2.6. The effect of the range of transmission angles of PWs on the CNR of reconstructed anechoic cysts at different depths in PW (dashed lines) and SAPW (solid lines) imaging methods. 

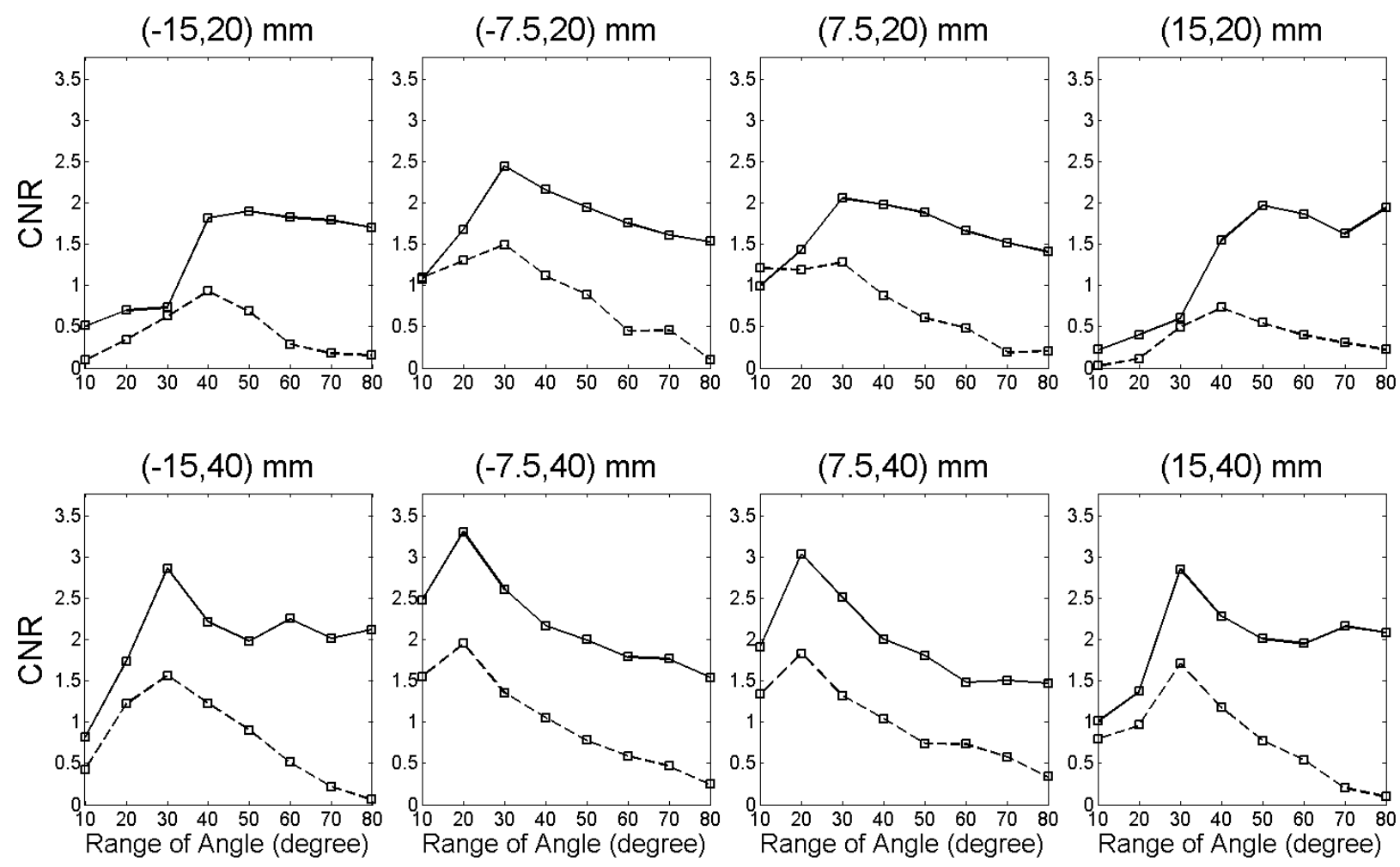

Fig. 2.7. The effect of the range of transmission angles of PWs on the CNR of reconstructed anechoic cysts at different lateral locations of PW (dashed lines) and SAPW (solid lines) imaging methods.

\section{B. Dynamic Apodization}

Figure 2.8 shows the effect of applying dynamic apodization on SAPW imaging using the optimal configuration of PW transmissions (19 with a step-size of 1.80 degrees of PW insonifications). Dynamic apodization for SAPW was applied in both transmitting and receiving with the same sub-aperture angles as the transmission angle. However, the dynamic apodization can only be applied in receiving with PW. Because of applying dynamic apodization, the advantage of increasing field of view has been reduced; however, the depth of penetration has been shown to improve (Figure 2.8). Reducing field of view happens naturally due to the calculation of dynamic apodization. It was reduced because the outside of the sub-aperture angle would not be used for reconstructing the image. Dynamic apodization increases the signal 
amplitude at large depth (Figure 2.9); however, it does not improve CNR directly (Figure 2.10). This effect will bring practical advantage when electric noise is added.
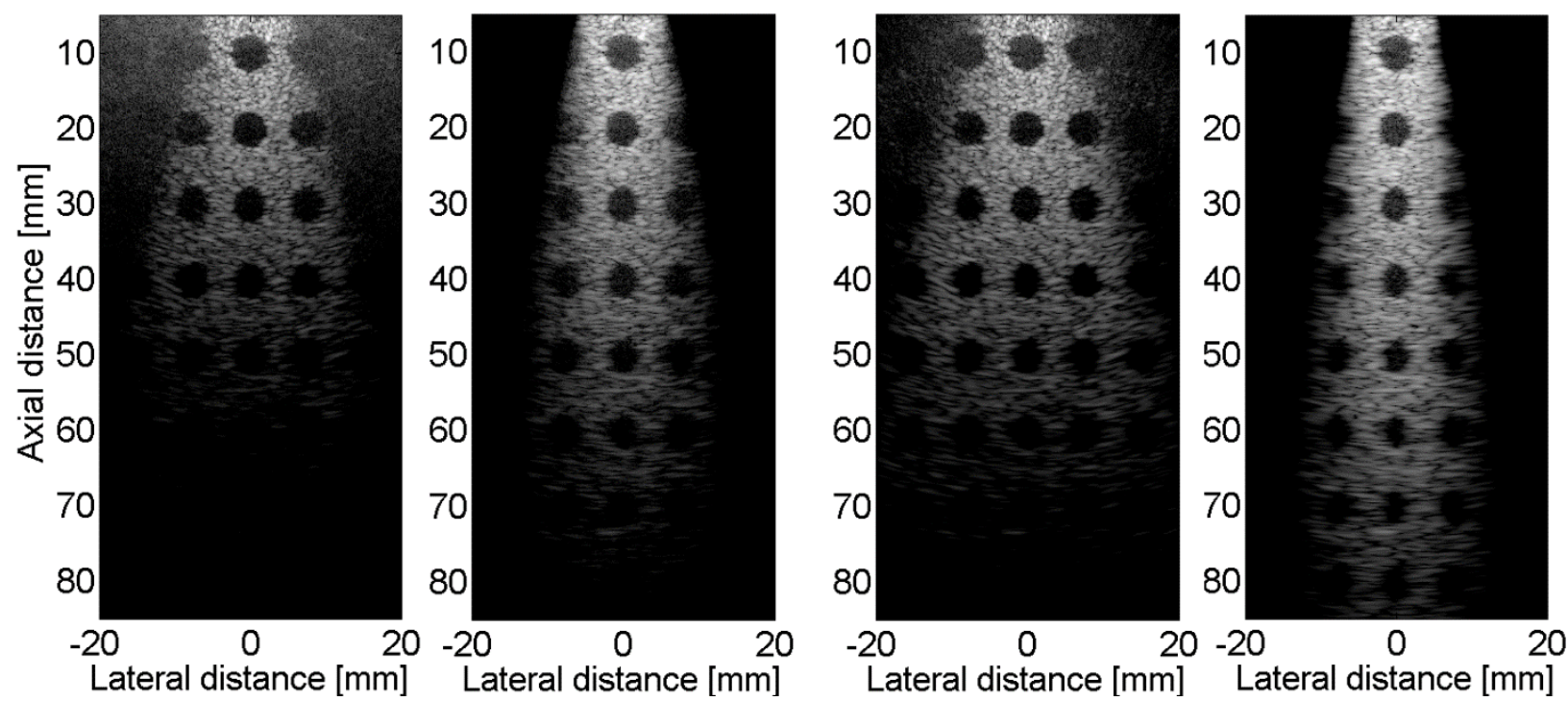

Fig. 2.8. Simulated log-enveloped beamformed PW without dynamic apodization (from left), PW with dynamic apodization in receiving, SAPW without dynamic apodization and SAPW with dynamic apodization in both transmitting and receiving imaging using the optimal configuration of PW in tissue mimicking phantom. 


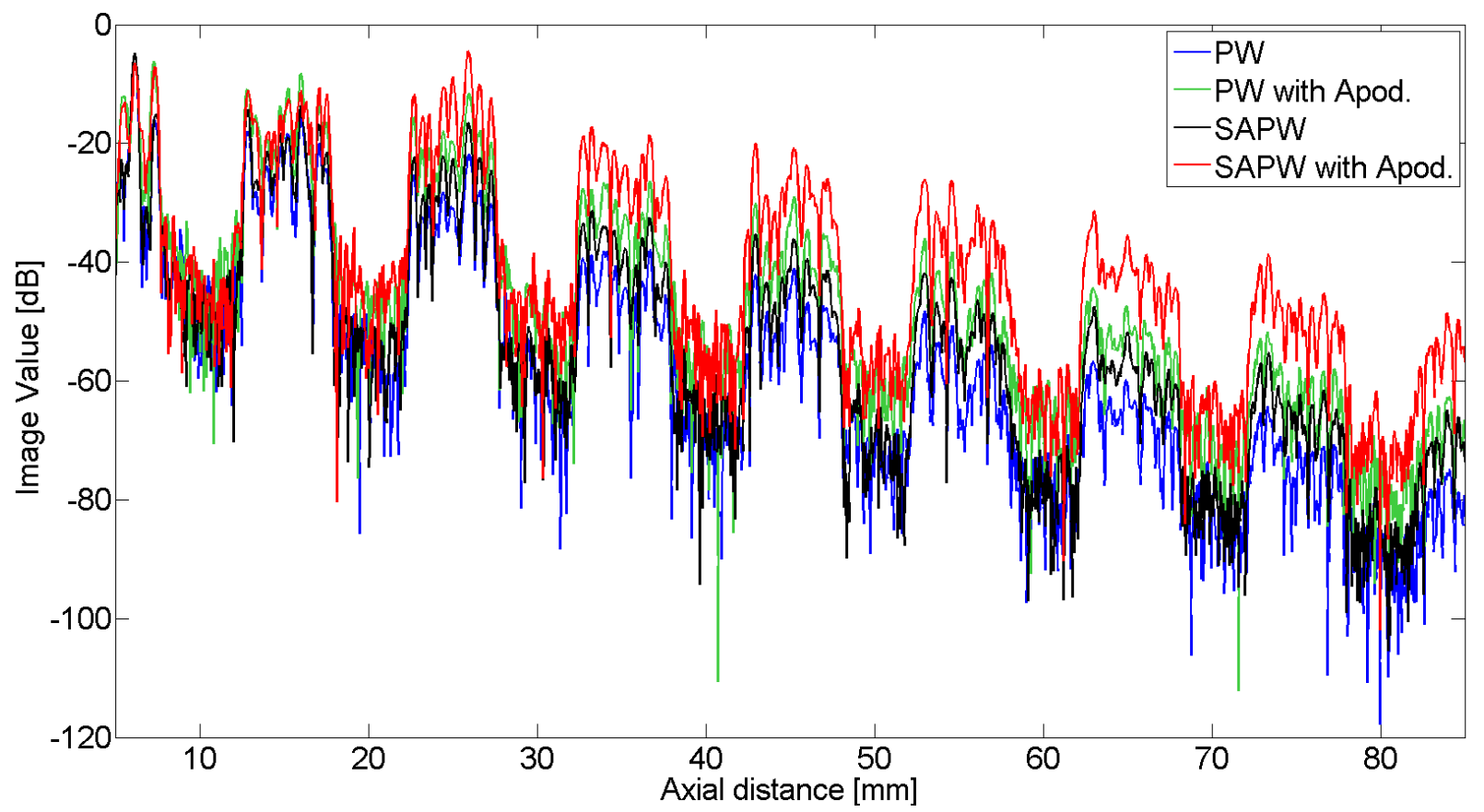

Fig. 2.9. Line profile comparison between PW and SAPW without and with dynamic apodization of Fig. 2.8 at different depth at the $0 \mathrm{~mm}$ lateral distance.

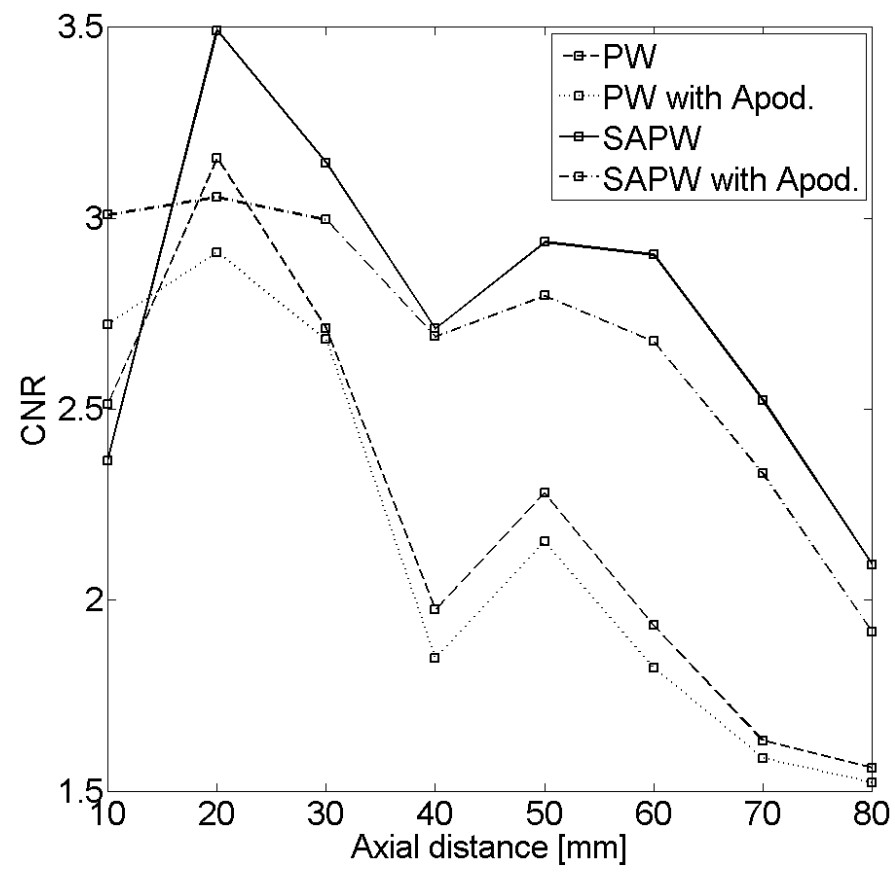

Fig. 2.10. CNR comparison between PW and SAPW without and with dynamic apodization corresponding to Fig. 2.8 at different depths and at the $0 \mathrm{~mm}$ lateral distance. 


\section{Singular Value Distribution of PW Transmission}

For all these simulation, a cut-off number of 1 was chosen. Moreover, the singular value distribution is different when frequency varies. Figure 2.11 shows the singular value distribution at three different frequencies when the PW RF data is obtained using the optimal configuration.
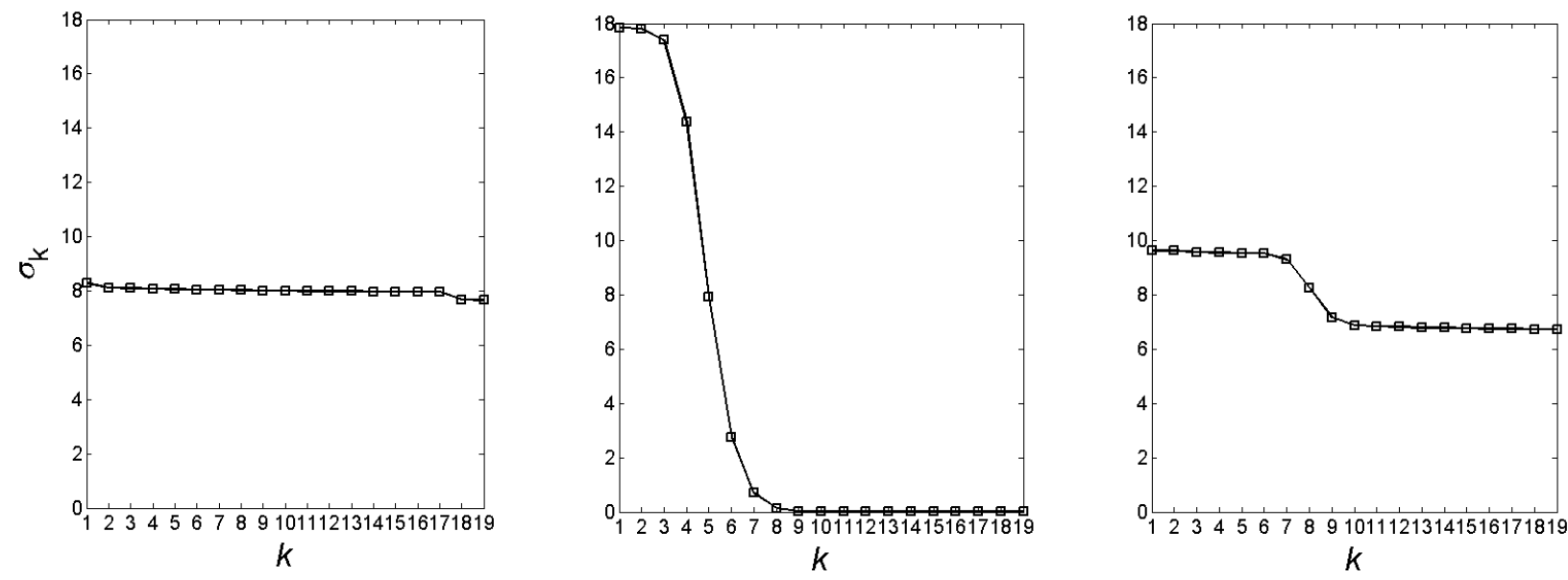

Fig. 2.11. The singular value distributions at three different frequencies with optimal configuration of $\mathrm{PW}$ imaging: central frequency, $5.0 \mathrm{MHz}$ (left), $1.0 \mathrm{MHz}$ (centre), and 7.0 MHz (right).

Since there were $19 \mathrm{PW}$ transmissions in optimal configurations, there are 19 singular values. Because the RF data at the central frequency is the most stable one, all singular values are evenly distributed. However, as frequency gets further away from the central frequency, the distributions have steeper change.

Most of the singular values near the central frequency tend to have a similar number because it gives the most stable RF data. For this simulation, at the central frequency with a range of the transmission angle of \pm 60 degrees, the first singular value was 8.80 and the last singular value was 7.23. Moreover, as the frequency gets farther away from the central frequency, the range of the singular values of those spectra gets wider and contains very low numbers. For example, at $1.00 \mathrm{MHz}$, the first singular value was 10.68 and the last singular value was 0.15 . 
Therefore, the cut-off number would filter values mostly from the contribution of further frequencies from the central frequency. Figure 2.12 shows the singular value distribution with PW transmission angles of \pm 60 degrees.
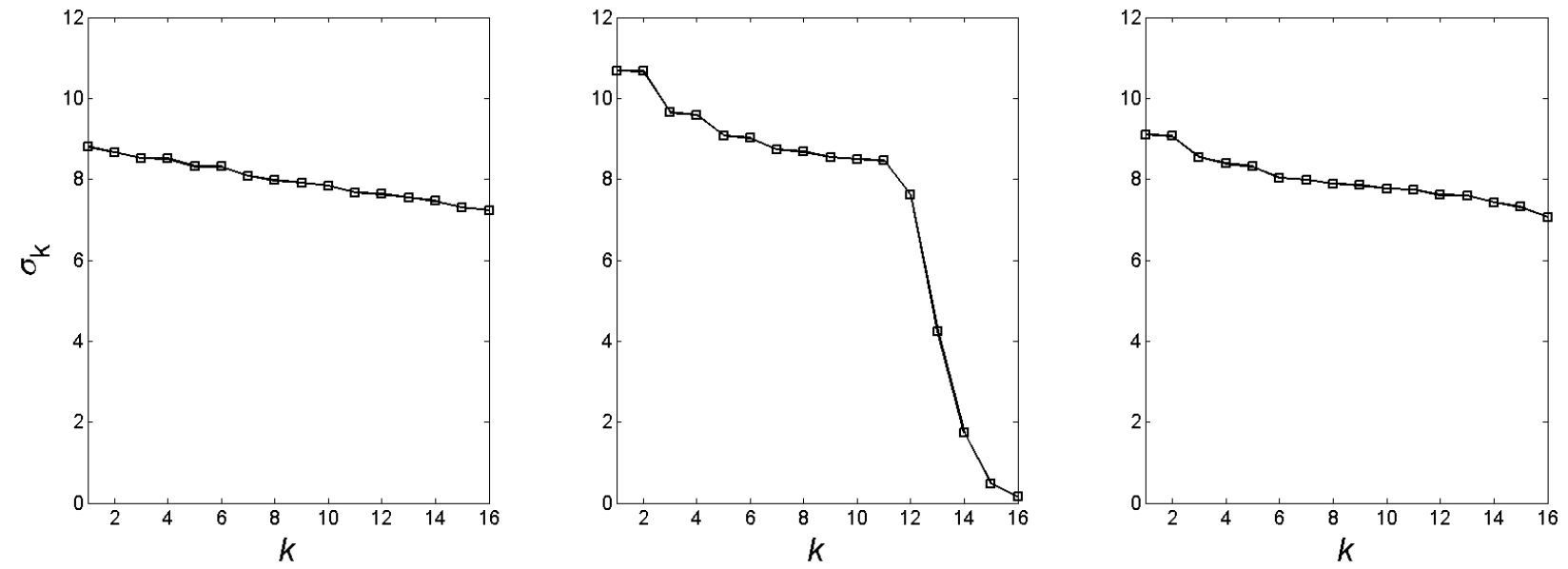

Fig. 2.12. Singular value distributions at three different frequencies with PW transmission angles of \pm 60 degrees: central frequency of $5.0 \mathrm{MHz}$ (left), $1.0 \mathrm{MHz}$ (centre), and $7.0 \mathrm{MHz}$ (right).

D. Noise

Figure 2.13 and 2.14 shows results of PW imaging and SAPW imaging in simulation with $1 \mathrm{~dB}$ white Gaussian noise added to RF signal. Due to noise, the depth of penetration has been reduced. As with the previous simulation, SAPW imaging also has improved depth of penetration and field of view in comparison to PW imaging. 

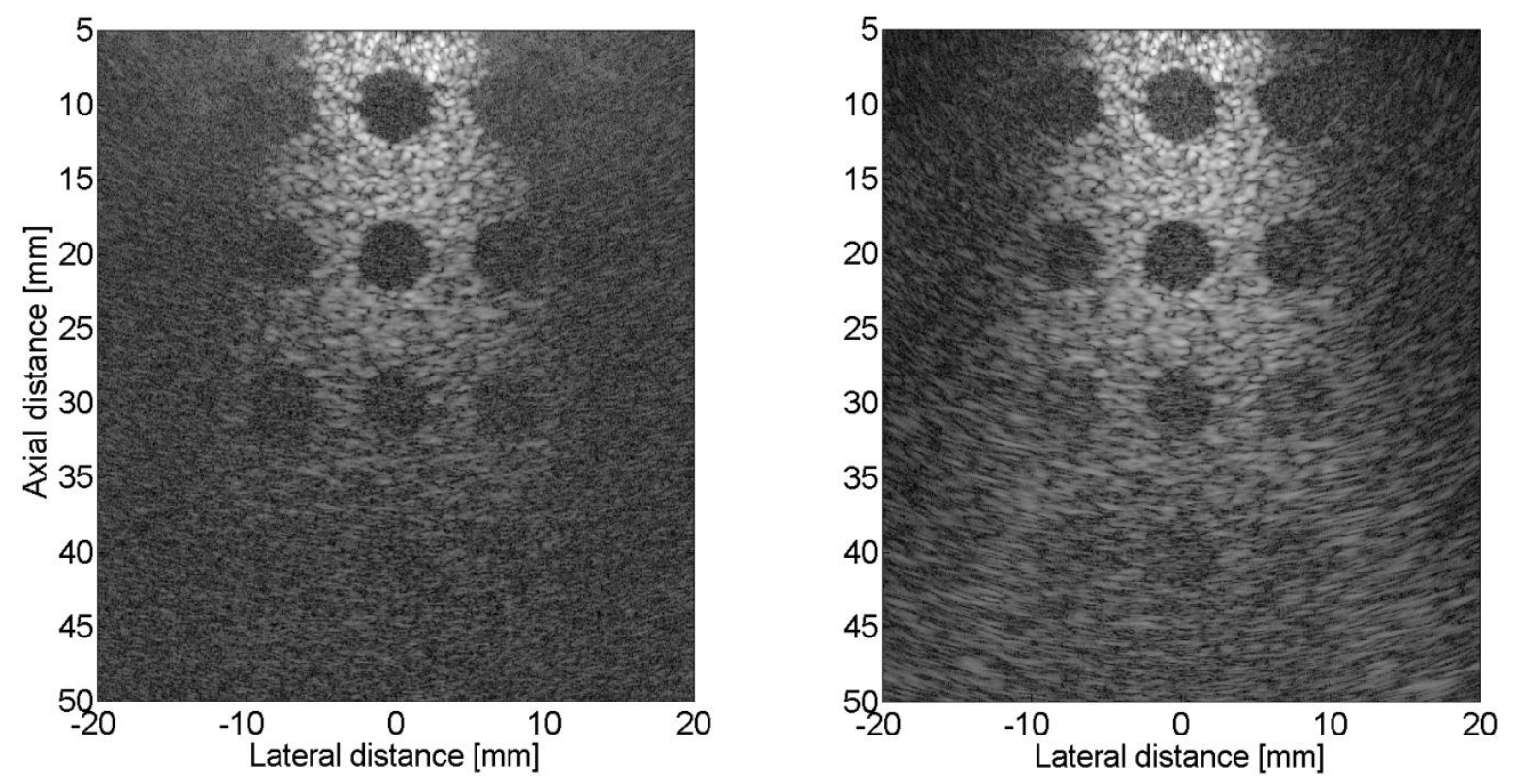

Fig. 2.13. Simulated log-enveloped beamformed PW (left) and SAPW (right) imaging using the optimal configuration of PW and $1 \mathrm{~dB}$ white Gaussian noise added to RF signal.
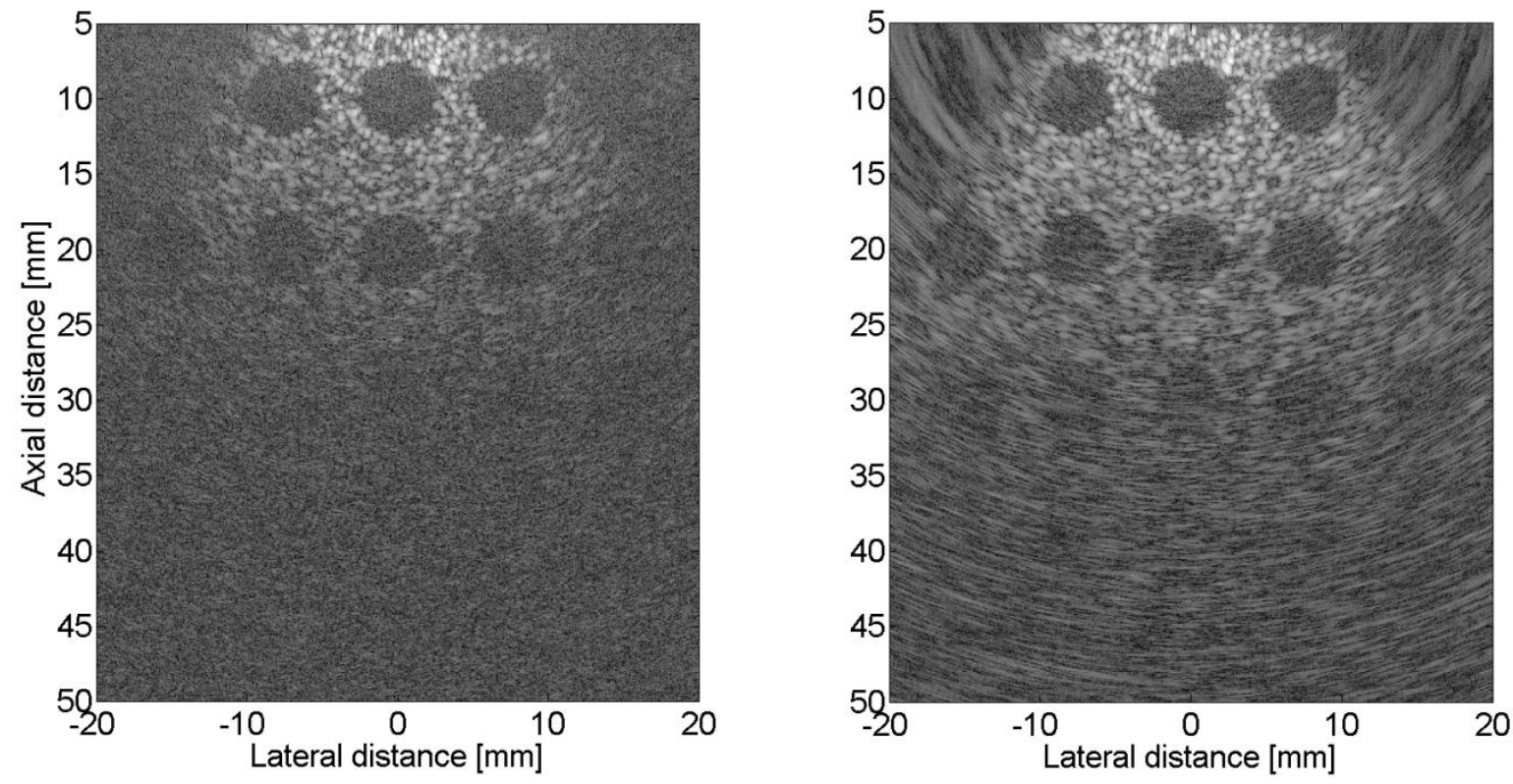

Fig. 2.14. Simulated log-enveloped beamformed PW (left) and SAPW (right) imaging in tissue mimicking phantom with $16 \mathrm{PW}$ transmissions, transmission angles of \pm 40 degrees and $1 \mathrm{~dB}$ white Gaussian noise added to RF signal. 


\section{E. Spatial Resolutions}

Figure 2.15 and 2.16 show the point scatter images with different ranges of transmission angles with the same number of transmissions in both PW and SAPW respectively. With visual inspection, the depth of penetration and field of view of SAPW imaging show improvements again; however, there is no significant improvement in spatial resolution in SAPW.
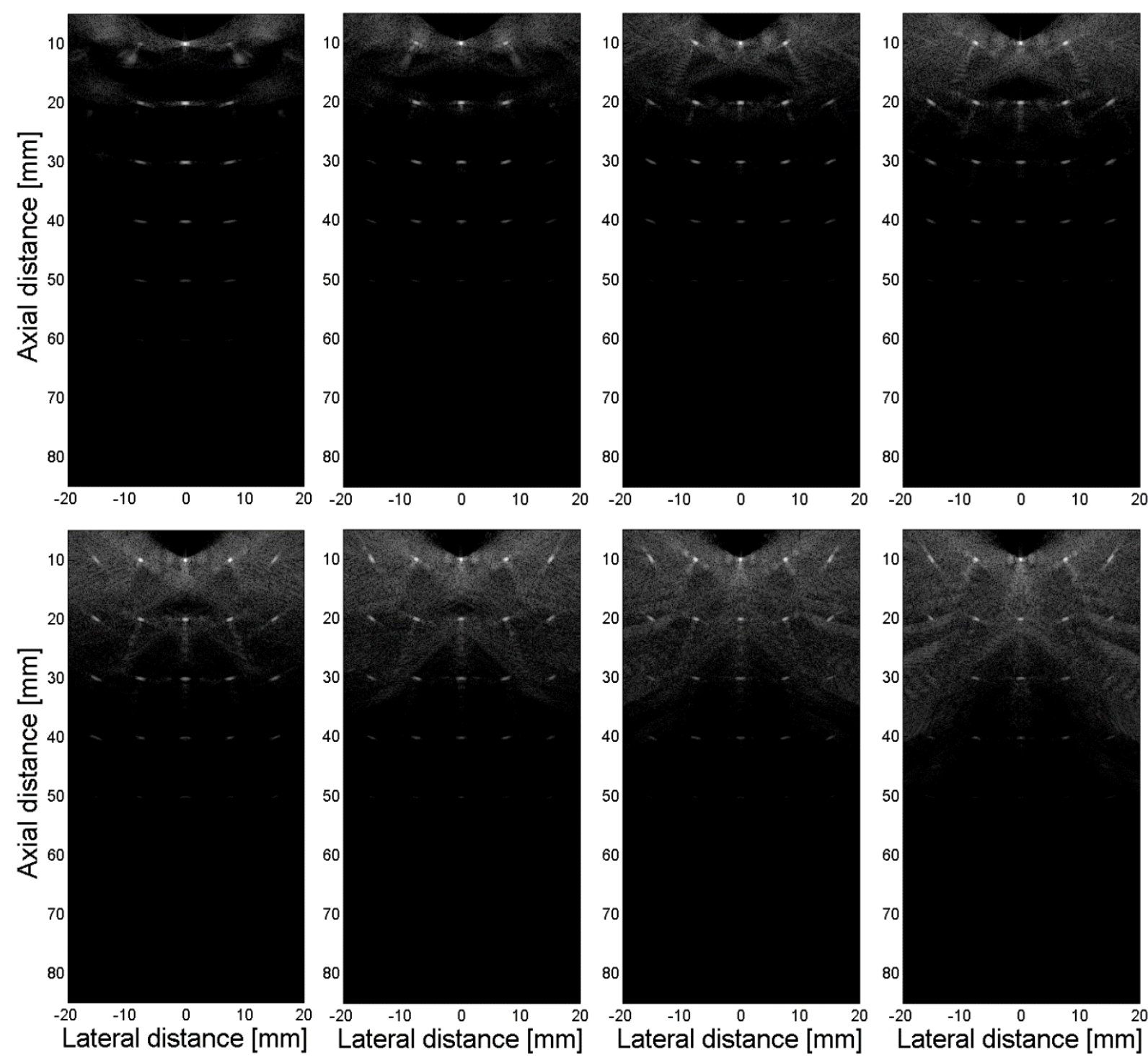

Fig. 2.15. Simulated log-enveloped beamformed PW imaging of point scatters with 16 transmissions and different range of angles. Top row: $\pm 10, \pm 20, \pm 30$ and \pm 40 degrees from left. Bottom row: $\pm 50, \pm 60, \pm 70$ and \pm 80 degrees from left. 

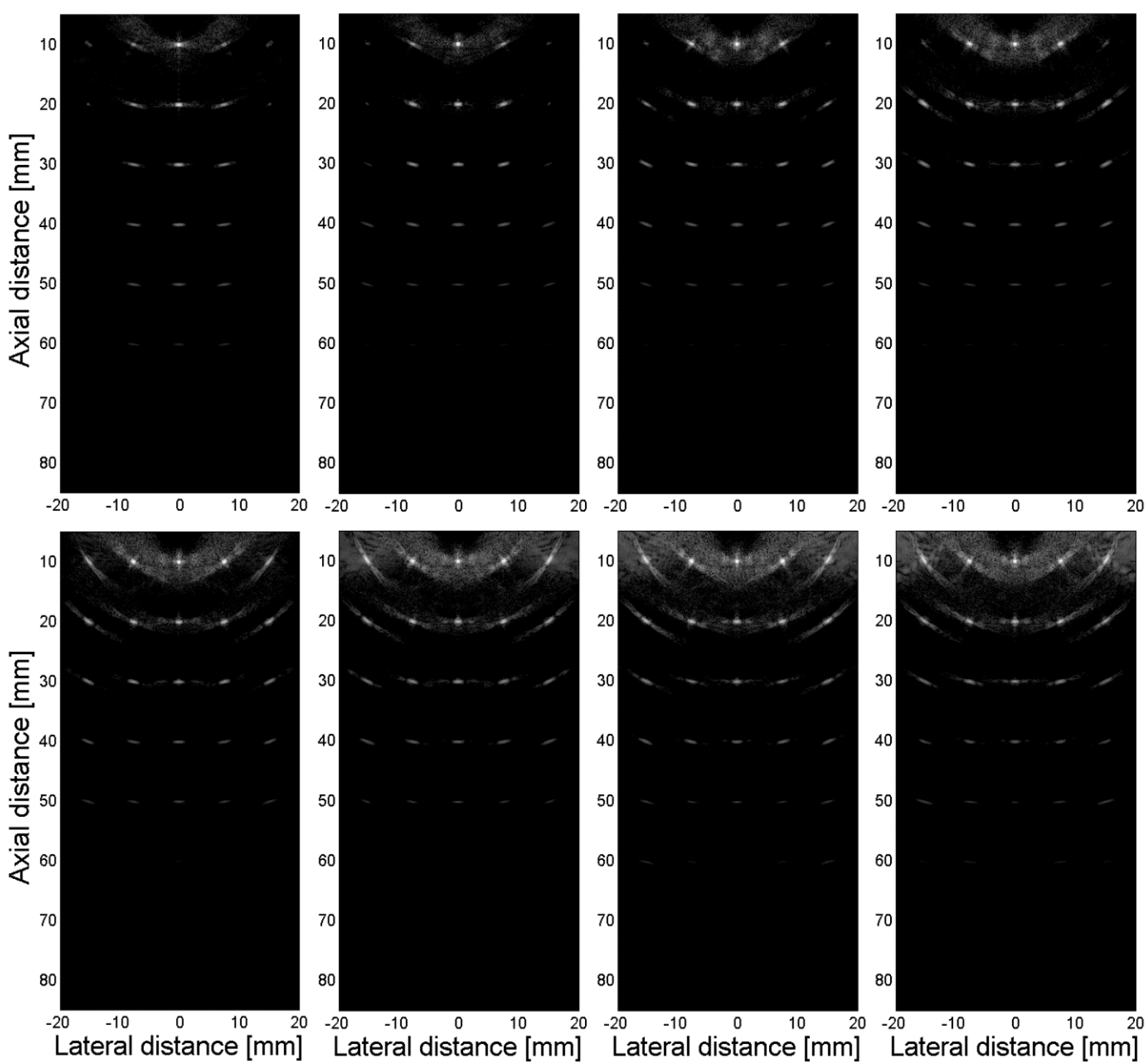

Fig. 2.16. Simulated log-enveloped beamformed SAPW imaging of point scatters with 16 transmissions and different range of angles. Top row: $\pm 10, \pm 20, \pm 30$ and \pm 40 degrees from left. Bottom row: $\pm 50, \pm 60, \pm 70$ and \pm 80 degrees from left.

The comparison between PW and SAPW imaging methods were conducted in a simulation study. Figures 2.15 and 2.16 show simulated log-enveloped beamformed images of point scatters with 16 transmissions and different range of angles from \pm 10 to \pm 80 degrees using PW imaging and SAPW imaging, respectively. There is no significant and consistent improvement in spatial resolution with the proposed SAPW imaging method over the PW imaging method as it observed 
from these simulation results. Figures 2.17 to 2.20 show the change in spatial resolution with the range of transmission angles at different locations for both imaging methods.
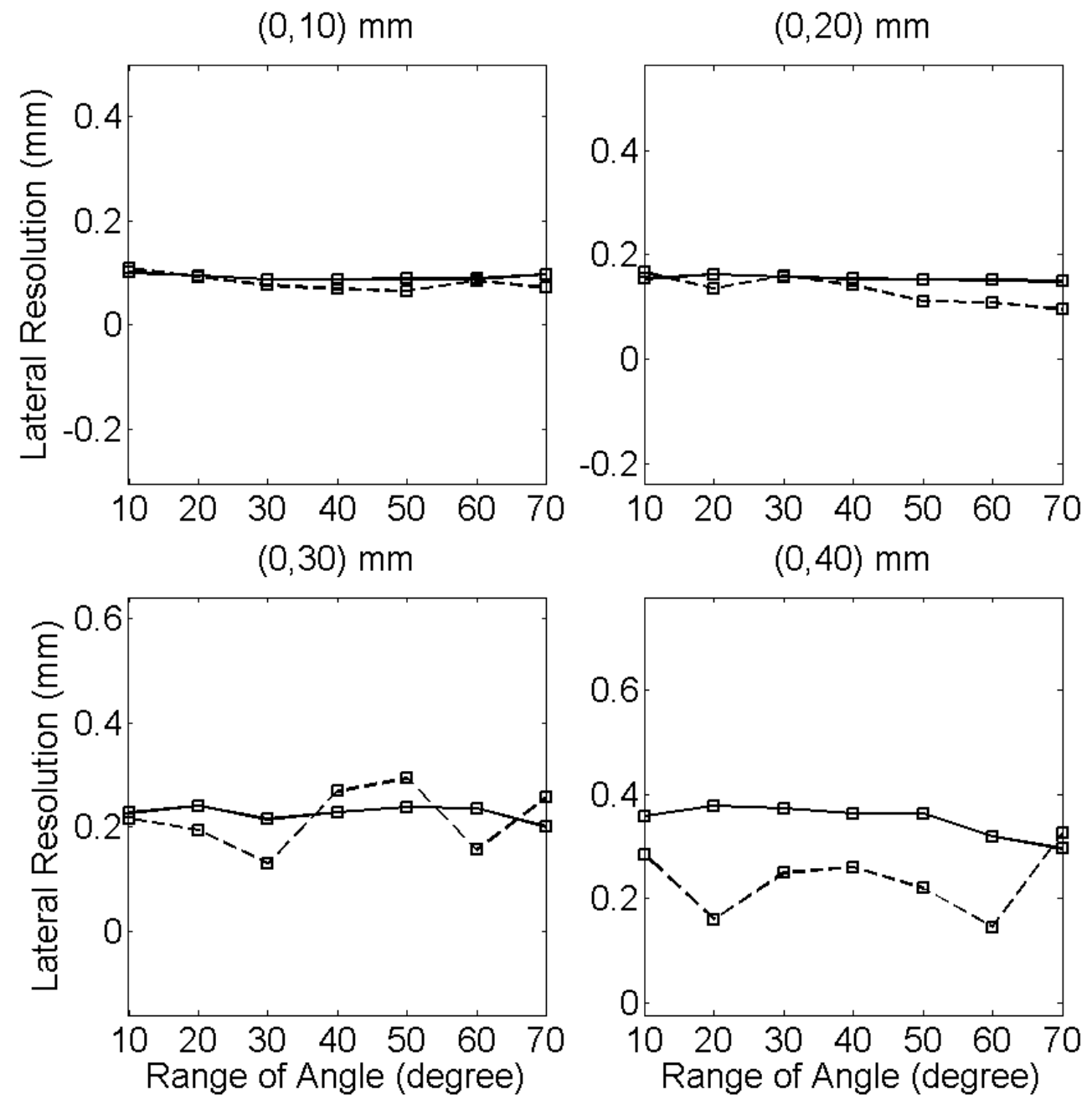

Fig. 2.17. The performance of the range of transmission angles with constant 16 transmission PWs and its effect on lateral resolution at different depths of PW (dashed lines) and SAPW (solid lines) imaging methods. 

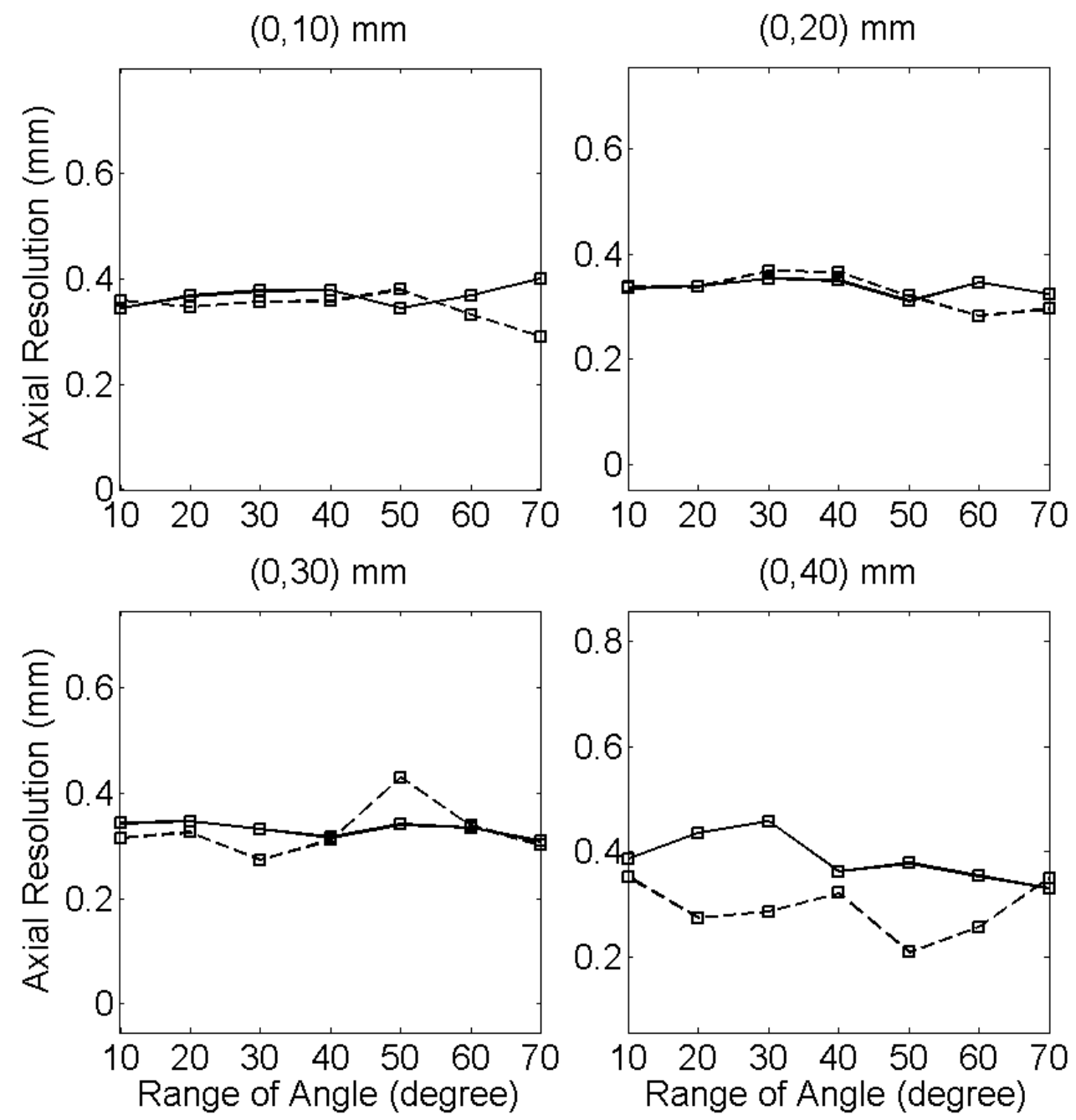

Fig. 2.18. The performance of the range of transmission angles with 16 transmission PWs and its effect on the axial resolution at different depths of PW (dashed lines) and SAPW (solid lines) imaging methods. 

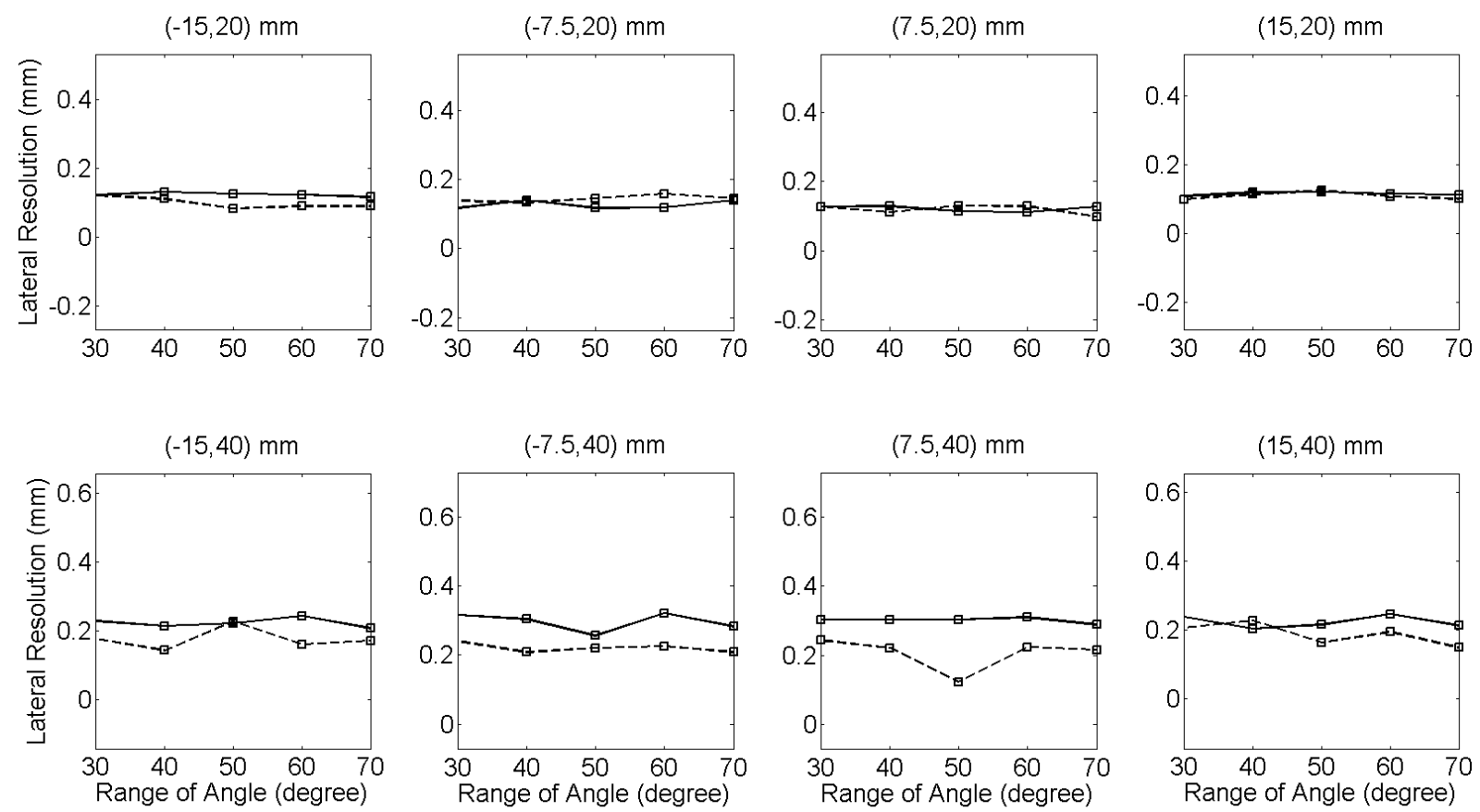

Fig. 2.19. The performance of the range of transmission angles with 16 transmission PWs and its effect on lateral resolution at different lateral distances of PW (dashed lines) and SAPW (solid lines) imaging methods. 

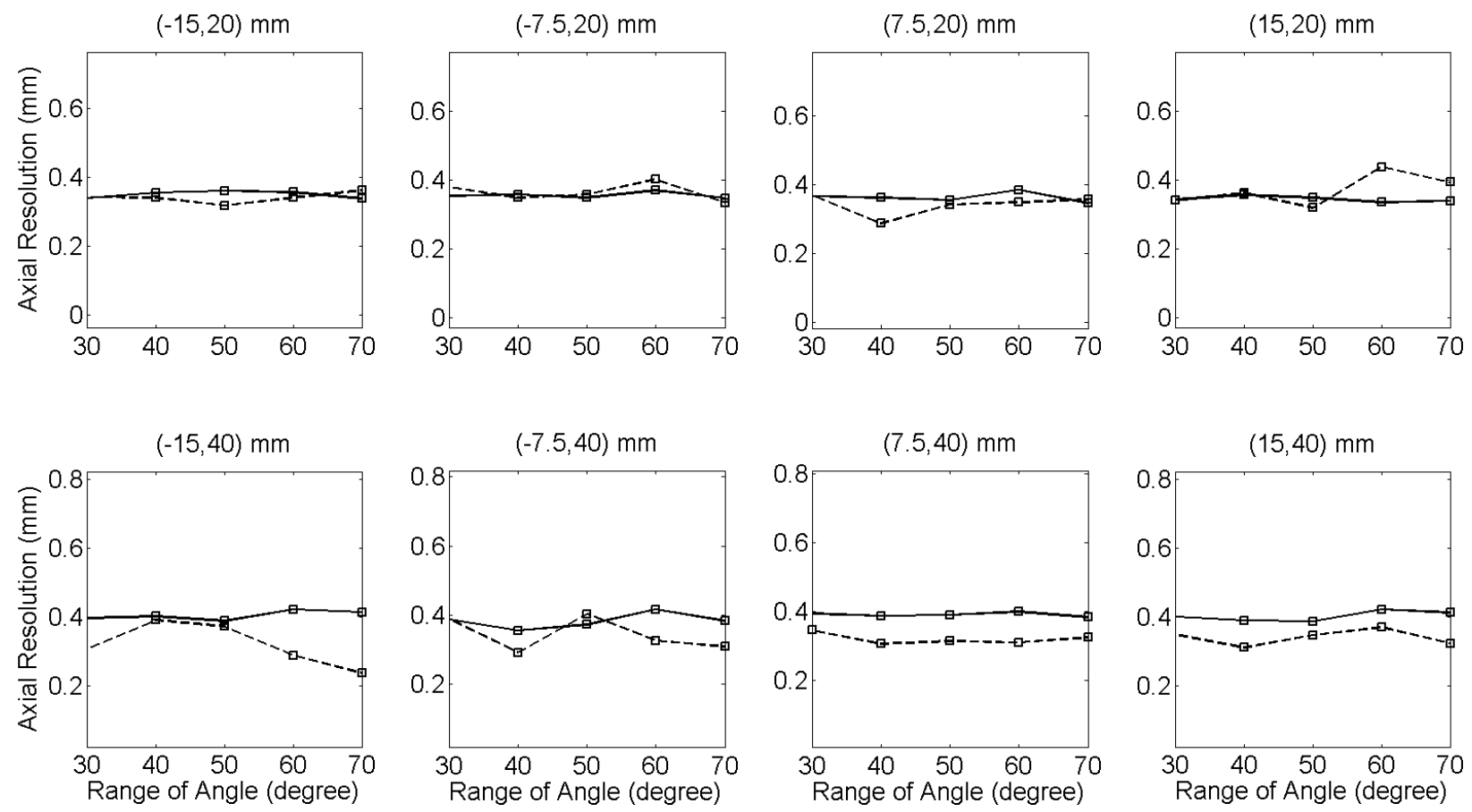

Fig. 2.20. The performance of the range of transmission angles with 16 transmission PWs and its effect on the axial resolution at different lateral distances of PW (dashed lines) and SAPW (solid lines) imaging methods.

\section{F. Summary and Discussion}

The improved results of SAPW over PW are the increase in depth of penetration and field of view. PW imaging suffers from limited depth of penetration due to lack of transmit focusing [16]. The assumption of the infinite extent of PWs fails at large depth even for PWs steered at a small angle. SAPW decodes delay-encoded matrix to estimate the RF signal that is equivalent to synthetic aperture imaging. Through the process of image reconstruction and combining low resolution images, fully dynamic focusing has been performed for all points in the image [8] without assuming an infinite extent of PWs. Since the transmitting and receiving focus at all points in the image have been made in SAPW, the depth of penetration of SAPW is better than PW.

The main advantage of using PW imaging is ultrafast frame rates. The data acquisition speed of SAPW is the same as PW imaging. However, the image reconstruction is more time- 
consuming in SAPW than PW due to the decoding process and reconstructing more low resolution images. The time it took for image processing and reconstruction in PW imaging and SAPW imaging was 85.46 seconds and 224.76 seconds, respectively in this study, using the parameters that were studied and using MATLAB R2013a. The configuration of the computer processor and memory was Intel® Core $^{\mathrm{TM}} \mathrm{i} 7-4770 \mathrm{~K}$ CPU operating at $3.50 \mathrm{GHz}$ (8 CPUs) and 16 GB RAM. The decoding processes for each frequency and each receiving channel are factors that affect data acquisition speed. After decoding, the image reconstruction in traditional STA imaging can be implemented in real time [17], [18]. Therefore, future work may focus on the optimization of SAPW imaging to speed up decoding.

\section{CONCLUSIONS}

This paper presents a new image reconstruction method (SAPW) to be used in conjunction with the PW RF signal ultrasound imaging technique. Using a unique delay sequence of PW transmissions, the RF signal contribution of individual elements was estimated using a pseudoinverse method based on the singular value decomposition technique. The new SAPW algorithm was simulated and evaluated using the Field II software. The estimated SAPW RF signal was stabilized by suppressing singular values below a cut-off value. The cut-off value was chosen in order to suppress the amplification of noise generated by small singular values in the proposed algorithm. To this end, the complete range of singular value components at the central frequency of the transmitted beam was investigated towards obtaining the most stable RF data. The results obtained demonstrate significant improvements in both field of view and depth of penetration in SAPW imaging compared to conventional PW imaging. Moreover, the depth of penetration has greater improvement when applying dynamic apodization. The field of view is naturally reduced due to the calculation of dynamic apodization. Dynamic apodization improves the depth of field by increasing the signal amplitude, but not CNR, directly at large depth. As the range of transmission angles increases, both PW and SAPW imaging methods show improvements in their respective field of views. However, the image quality of SAPW imaging improves at a higher rate compared to image quality of PW imaging by widening the range of 
angles, increasing the angular step-size, and lowering the number of transmissions. There is no significant and consistent improvement in spatial resolution with the proposed SAPW imaging method over the PW method as observed from these simulation results. This study can be extended further to other array transducer geometries. An important practical example is the convex array which typically has a low depth of penetration due to its diverging beam geometry. Since the proposed SAPW method increases the depth of penetration, it can potentially improve the image quality of convex array transducers. This simulation study was entirely conducted using the Field II software [13], [14] and a linear phased array transducer. The linear phased array transducer was used due to its large range of steering angles. In the next phase of the study, an experimental validation of the SAPW method will be conducted using a research ultrasound imaging system.

\section{ACKNOWLEDGMENTS}

The authors would like to thank Ying Li from Department of Physics, Ryerson University, for his technical help in this study. Research supports for this study were provided by Natural Sciences and Engineering Research Council of Canada discovery grants that were awarded to Drs. Y. Xu and J. Tavakkoli. 


\section{REFERENCES FOR CHAPTER 2}

[1] T. L. Szabo, “Diagnostic Ultrasound Imaging: Inside Out”, Academic Press, 2014.

[2] L. Sandrin, S. Catheline, M. Tanter, X. Hennequin, and M. Fink, "Time resolved pulsed elastography with ultrafast ultrasonic imaging," Ultrason. Imaging, vol. 21, pp. 259-272, Oct. 1999.

[3] L. Sandrin, S. Catheline, M. Tanter, C. Vinconneau, and M. Fink, "2D transient elastography," Acoust. Imaging, vol. 25, pp. 485-492, Jan. 2000.

[4] L. Sandrin, M. Tanter, S. Catheline, and M. Fink, "Shear modulus imaging using 2D transient elastography," IEEE Trans. Ultrason. Ferroelectr. Freq. Control, vol. 49, no. 4, pp. 426-435, Apr. 2002.

[5] G. Montaldo, M. Tanter, J. Bercoff, N. Benech, and M. Fink, "Coherent planewave compounding for very high frame rate ultrasonography and transient elastography." IEEE Transactions on Ultrason. Ferroelectr. Freq. Control, vol. 56, pp. 489-506, 2009.

[6] J. Jensen, O. Holm, L. J. Jerisen, H. Bendsen, S. I. Nikolov, B. G. Tomov, P. Munk, M. Hansen, K. Salomonsen, J. Hansen, K. Gormsen, H. M. Pedersen, and K. L. Gammelmark, "Ultrasound research scanner for real-time synthetic aperture data acquisition," IEEE Trans. Ultrason. Ferroelectr. Freq. Control, vol. 52, no. 5, pp. 881-891, May 2005.

[7] T. Misaridis and J. A. Jensen, "Use of modulated excitation signals in medical ultrasound. Part III: High frame rate imaging," IEEE Trans. Ultrason. Ferroelectr. Freq. Control, vol. 52, no. 2, pp. 208-219, Feb. 2005.

[8] J. A. Jensen, S. I. Nikolov, K. L. Lokke, and M. H. Pedersen, "Synthetic aperture ultrasound imaging", Ultrasonics, vol. 44, pp.e5-e15, 2006 
[9] P. Gong, A. Moghimi, M. C. Kolios, and Y. Xu, "Delay-encoded Transmission in Synthetic Transmit Aperture (DE-STA) Imaging," IEEE International Ultrasonics Symposium (IUS), 2014.

[10] P. Gong, M. C. Kolios, and Y. Xu, "Delay-encoded Transmission and Image Reconstruction Method in Synthetic Transmit Aperture Imaging," IEEE Trans. Ultrasonics, Ferroelectrics and Frequency Control, 2015.

[11] P. Gong, M. C. Kolios, and Y. Xu, " Pseudo-Inverse (PI) Decoding process in Delayencoded Synthetic Transmit Aperture (DE-STA) Imaging," IEEE Trans. Ultrasonics, Ferroelectrics and Frequency Control, in press, 2015.

[12] H. Anton, and R. C. Busby, "Contemporary Linear Algebra”, John Wiley \& Sons, Inc., 2003

[13] J. A. Jensen and N. B. Svendsen, "Calculation of pressure fields from arbitrary shaped, apodized, and excited ultrasound transducers," IEEE Trans. Ultrason. Ferroelect. Freq. Contr., vol. 39, pp. 262-267, Mar. 1992.

[14] J. A. Jensen, "Field: A program for simulating ultrasound systems," Med. Biol. Eng. Comput., vol. 34, pp. 351-353, 1996.

[15] K. F. Üstüner and G. L. Holley, "Ultrasound imaging system performance assessment," presented at the 2003 American Association of Physicists in Medicine Annu. Meeting, San Diego, CA, Aug. 2003.

[16] P. Song, M. W. Urban, A. Manduca, J. F. Greenleaf, and S. Chen, "Coded Excitation Plane Wave Imaging for Shear Wave Motion Detection", IEEE Trans. Ultrason. Ferroelect. Freq. Contr., vol. 62, no. 7, pp. 1356-1372, Jul. 2015.

[17] J. A. Jensen, O. Holm, L. J. Jensen, H. Bendsen, S. I. Nikolov, B. G. Tomov, P. Munk, M. Hansen, K. Salomonsen, J. Hansen, K. Gormsen, H. M. Pedersen and K. L. Gammelmark, 
"Ultrasoundresearch scanner for real-time synthetic aperture data acquisition," IEEE Trans. Ultrason. Ferroelect. Freq. Contr., vol. 52, pp. 881-891, May 2005.

[18] J. A. Jensen, H. Holten-Lund, R. T. Nilsson, M. Hansen, U. D. Larsen, R. P. Domsten, B. G. Tomov, M. B. Stuart, S. I. Nikolov, M. J. Pihl, Y. Du, J. H. Rasmussen and M. F. Rasmussen, "SARUS: A Synthetic Aperture Real-Time Ultrasound System," IEEE Trans. Ultrason. Ferroelect. Freq. Contr., vol. 60, pp. 1838-1852, Sep. 2013. 


\section{Chapter 3}

\section{Conclusions and Suggestions for Future Work}

\subsection{Summary}

The proposed image reconstruction method for PW RF data was defined as SAPW (synthetic-aperture plane wave). It simulates the RF data that is equivalent to the STA RF data through pseudoinverse using SVD. The RF data of the PW is converted from time domain to frequency domain using Fourier transform. Using a unique delay sequence of the PW transmissions, the RF signal contribution of individual elements which is the STA equivalent RF signal was calculated using a pseudoinverse method based on the singular value decomposition technique on the delay-encoded matrix. In order to stabilize the calculated RF data, the singular value threshold was introduced. It was concluded that the cut-off singular value should be less than the last singular value of the central frequency and greater or equal to 1 . All singular value components from the central frequency should be included because the RF data of the central frequency is the most stable data. Moreover, it should be greater than or equal to 1 to avoid any noise implication from the inversing the singular value smaller than 1 and greater than 0 . After the STA equivalent RF signal is calculated in time domain, the RF data is reconstructed using synthetic aperture image reconstruction method. The performance of SAPW was evaluated using CNR and spatial resolutions. The SAPW was compared with PW imaging reconstruction method and showed the improvements in the depth of penetration and the field of view; however, the spatial resolutions of SAPW did not show significant improvement. 


\subsection{Conclusions}

This thesis presents a new image reconstruction method to be used with the PW RF signal ultrasound imaging technique. The new SAPW algorithm was simulated and evaluated using Field II software and MATLAB R2013a. The advantages of using SAPW are: (a) ability to estimate the synthetic aperture imaging data with lower number of transmissions (typically the number of transmissions in synthetic aperture imaging is the same as the number of the elements), and (b) the actual contribution on PW by each element is calculated without assuming the wave front as an ideal PW that is an infinite extent PW, because spherical waves generated by individual elements are all considered in calculation.

The results obtained demonstrate significant improvements in both field of view and depth of penetration in SAPW imaging compared to conventional PW imaging. As the range of transmission angles increases, both PW and SAPW imaging methods show improvements in their respective field of views. However, the image quality of SAPW imaging can potentially compensate for the lowering image quality of PW imaging by widening the range of the angles, increasing the angular step-size, and lowering the number of the transmissions. For the spatial resolutions, there is no significant and consistent improvement in the proposed SAPW imaging method over the PW method as it is observed from these simulation results. For the temporal resolution, the SAPW imaging has $168 \%$ increase in simulation computing time in comparison to PW imaging. SAPW has shown the significant improvement on imaging a large area in deeper depth; therefore, SAPW imaging can compensate for fast temporal resolution of PW imaging.

\subsection{Suggestions for Future Works}

This study can further be extended to other array transducer geometries. An important practical example is the convex array which typically has a low depth of penetration due to its diverging beam geometry. Since the proposed SAPW method increases the depth of the penetration, it can potentially improve the image quality using the convex array transducers.

The simulations in this thesis were mostly conducted using the Field II software. A preliminary experimental validation of the SAPW is presented in Appendix A. The optimization 
of experiment result can be further investigated. Moreover, the PW with large range of angle requires large amount of delay which has so far been difficult to implement with current experimental setup. Moreover, PW imaging can be compared with other encoded transmissions such as Hadamard transmission which briefly studied in Appendix B. 


\section{Appendix A}

\section{A Preliminary Experimental Validation of the Synthetic-Aperture Plane Wave (SAPW)}

A preliminary experimental validation of the SAPW imaging method has been conducted in this study. The experimental data was acquired using a research imaging system (Vantage Platform, Verasonics Inc., Kirkland, WA, USA). Table A.1 shows the parameters that were used in the experiments. The array probe is a typical linear array that has 128 elements. Each element has width of $0.250 \mathrm{~mm}$ and pitch of $0.298 \mathrm{~mm}$. The central frequency of the array probe was 2.5 $\mathrm{MHz}$ and data was sampled at $10 \mathrm{MHz}$. A tissue mimicking phantom was used in the experiment. The phantom was made of degassed water (93.85\% of total weight), gelatin powder (4.69\%), polyethylene oxide (scatter) $(1 \%)$ and formaldehyde $(0.46 \%)$. In the background of the phantoms, the scatter concentration was $1 \%$ of the total weight, while inside the hypo-echoic inclusions located at depth of $37 \mathrm{~mm}$ and $85 \mathrm{~mm}$ had no scatters and the weak hyper-echoic inclusion located at depth $60 \mathrm{~mm}$ had the scatter concentration of $1.4 \%$. 
Table A.1. PW experiment parameters

\begin{tabular}{ll}
\hline Experiment Parameters & Default Values \\
\hline Central Frequency $\left(\mathrm{f}_{\mathrm{o}}\right)$ & $2.5 \mathrm{MHz}$ \\
Sampling Frequency $\left(\mathrm{f}_{\mathrm{s}}\right)$ & $10 \mathrm{MHz}$ \\
Number of Transmissions & 16 \\
Cut-off Parameter for SVF & 1 \\
Speed of sound $\left(\mathrm{c}_{\mathrm{o}}\right)$ & $1540 \mathrm{~m} / \mathrm{s}$ \\
Number of Elements & 128 \\
Wavelength $\left(\lambda_{\mathrm{o}}\right)$ & $0.616 \mathrm{~mm}$ \\
Array Element Pitch & $0.298 \mathrm{~mm}$ \\
Array Element Width & $0.250 \mathrm{~mm}$ \\
Dynamic Range of the Images & $60 \mathrm{~dB}$ \\
\hline
\end{tabular}

Figure A.1 and A.2 shows the experimental images of PW, SAPW and B-mode. B-mode image was taken with a focal depth at $60 \mathrm{~mm}$ where weak hyper-echoic inclusion is located. Figure A.1 was performed with $16 \mathrm{PW}$ transmissions and the angular range of transmissions of \pm 15.88 degrees and Figure A.2 was performed with 16 PW transmission and the angular range of transmissions of \pm 40 degrees

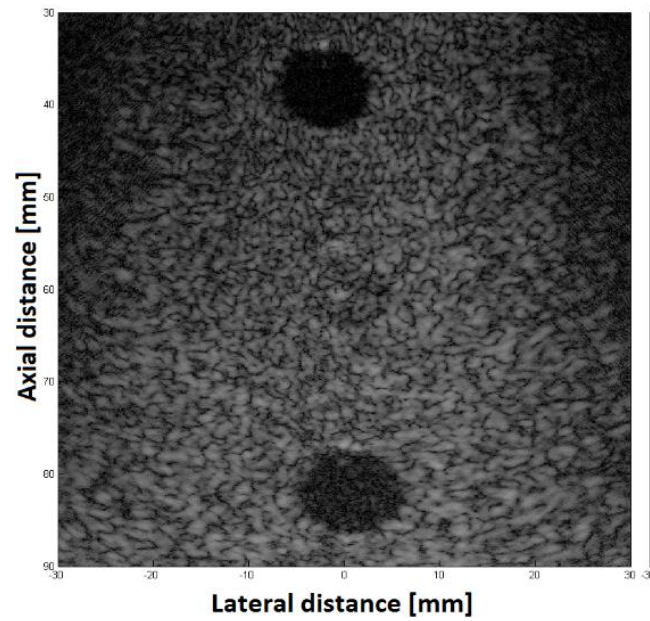

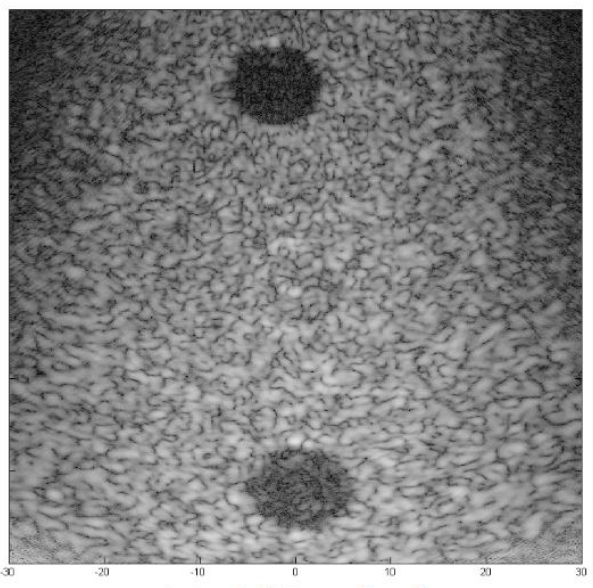

Lateral distance $[\mathrm{mm}]$

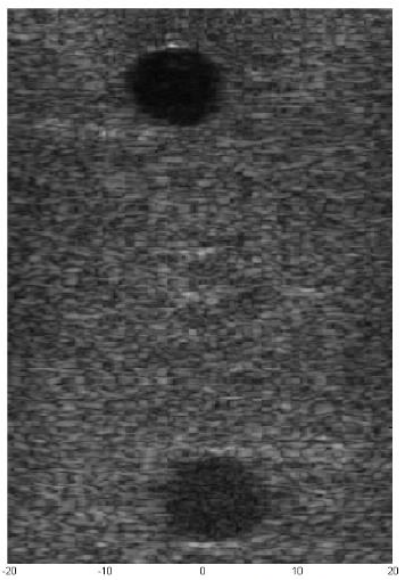

Lateral distance $[\mathrm{mm}]$

Fig. A.1. The experimental results of the tissue mimicking phantom in 16 transmission of PW with range of transmission of \pm 15.88 degrees. PW imaging (left,), SAPW imaging (middle) and conventional B-mode imaging (right) 


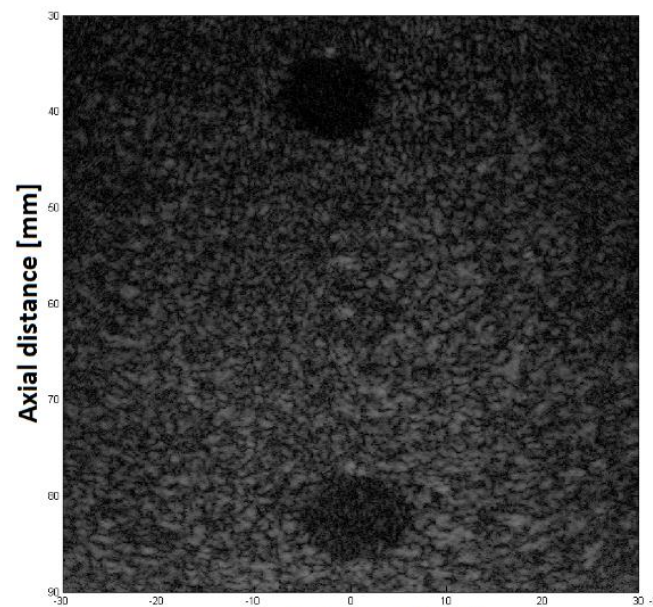

Lateral distance $[\mathrm{mm}]$

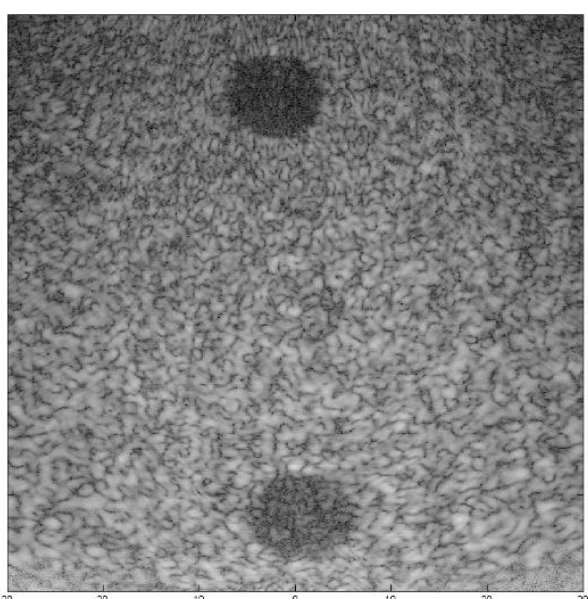

Lateral distance $[\mathrm{mm}]$

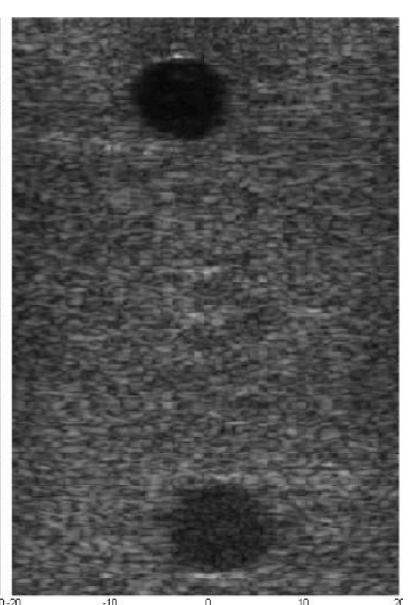

Lateral distance $[\mathrm{mm}]$

Fig. A.2. The experimental results of the tissue mimicking phantom in 16 transmission of PW with range of transmission of \pm 40 degrees. PW imaging (left,), SAPW imaging (middle) and conventional B-mode imaging (right)

Table A.2. Preliminary experimental quantitative comparison for the different methods.

\begin{tabular}{lllll}
\hline \multirow{2}{*}{ Imaging mode } & $\begin{array}{l}\text { CNR } \\
\text { (Depth 37 mm, } \\
\text { Experiment) }\end{array}$ & $\begin{array}{l}\text { CNR } \\
\text { (Depth 37 mm, } \\
\text { Simulation) }\end{array}$ & $\begin{array}{l}\text { CNR } \\
\text { (Depth 85 mm, } \\
\text { Experiment) }\end{array}$ & $\begin{array}{l}\text { CNR } \\
\text { (Depth 85 mm, } \\
\text { Simulation) }\end{array}$ \\
\hline B-mode & 2.98 & 3.03 & 1.74 & 1.77 \\
PW $\left( \pm 15.88^{\circ}\right)$ & 2.03 & 1.97 & 1.82 & 1.84 \\
SAPW $\left( \pm 15.88^{\circ}\right)$ & 2.13 & 2.71 & 2.16 & 2.40 \\
PW $\left( \pm 40^{\circ}\right)$ & 0.93 & 0.91 & 1.05 & 0.93 \\
SAPW $\left( \pm 40^{\circ}\right)$ & 1.39 & 1.94 & 1.52 & 1.73 \\
\hline
\end{tabular}

The preliminary experiment results matches with the simulation studies. SAPW shows better improvement at the deeper depth. Also, the SAPW shows better improvement when the step size of the transmission angle increases. Since this is just a preliminary result, the SAPW image can further be improved and optimized in the future phase of this study. Moreover, both PW and SAPW detects the weak hyper-inclusion located in the depth of $60 \mathrm{~mm}$. 


\section{Appendix B}

\section{Comparison between Hadamard Encoded Transmission and Specifically Reduced Transmission}

Hadamard encoded transmission imaging is known as the best standard method to improve the low signal-to-noise limitation of synthetic aperture imaging by generating a field of much higher energy. [1]-[3] In this Hadamard encode transmission, a part of the array elements transmit a pulse (positive pulse) while the others transmit a phase inverted version (negative pulse) of the same pulse simultaneously. The sequence and order of the pulses is determined by the coding matrix called Hadamard matrix which named after the French mathematician Jacques Hadamard. This matrix is a square matrix which composes of either +1 or -1 and whose rows are mutually orthogonal. Each row of the Hadamard matrix would determine which pulse to transmit. +1 is corresponded to positive pulse. On the other hand, -1 is corresponded to a negative pulse. After multiple transmissions, the backscatter signals received from all the transmissions can be processed to obtain the traditional STA data. However, Hadamard encoded transmission requires $n$ (number of elements of a probe) number of transmissions to have the best results. [4] In this study, a multiple of quarter transmission of Hadamard encoded transmission imaging is specifically chosen. It will be compared to the SAPW imaging that had a same number of transmissions; in this case, it will be 16 transmissions with same simulation parameter. From 64 Hadamard encoded transmission, 8 different sequences of transmissions (Rows of Hadamard matrix) were chosen: $r, r+16, r+32, r+48,4 r-3,4 r-2,4 r-1$ and $4 r$ where $r=Z$ $\epsilon[1,16]$. Figure B.1 shows the simulated log-enveloped image of the anechoic soft tissue phantom by 8 different Hadamard sequences of transmissions. 

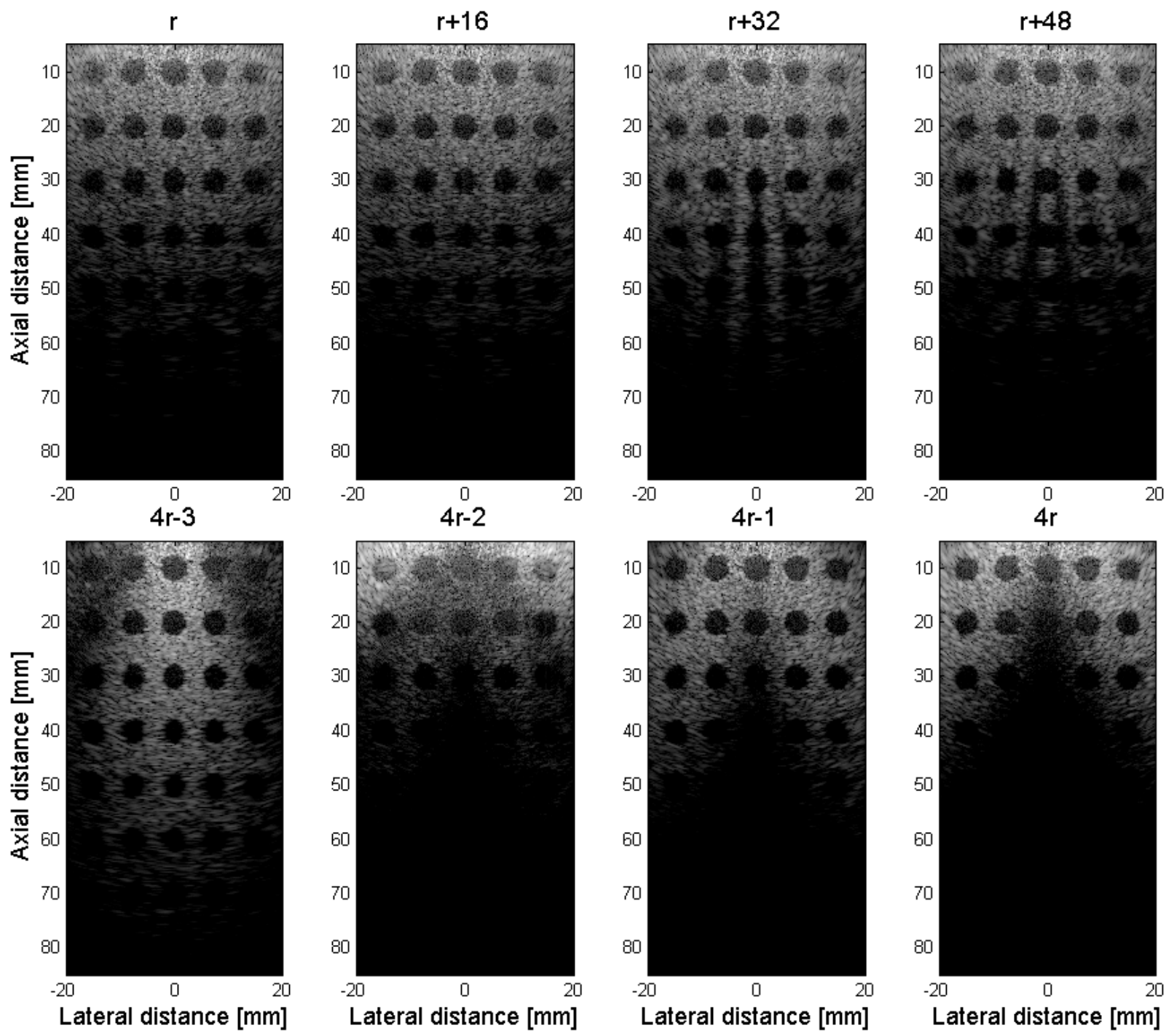

Fig. B.1. Simulated log-enveloped beamformed imaging of anechoic soft tissue phantom with 16 transmissions with different sequences of the quarter of Hadamard encoded transmissions.

r, r+16, r+32, r+48, 4r-3 Quarter Hadamard encoded transmission imaging also shows the increases in the field of view and the depth of penetration than the PW imaging. The interesting results of quarter Hadamard encoded transmission imaging is the results of 4r-2, 4r-1 and 4r. The imaging result shows that there is strong detection capability in the region outside of the aperture than the region under the aperture. If the region under the aperture is the region of the interest, 
the transmission 4r-3 shows the best CNR result. Figure B.2 shows the CNR comparison of different quarter Hadamard transmission and SAPW of anechoic inclusions at different location.

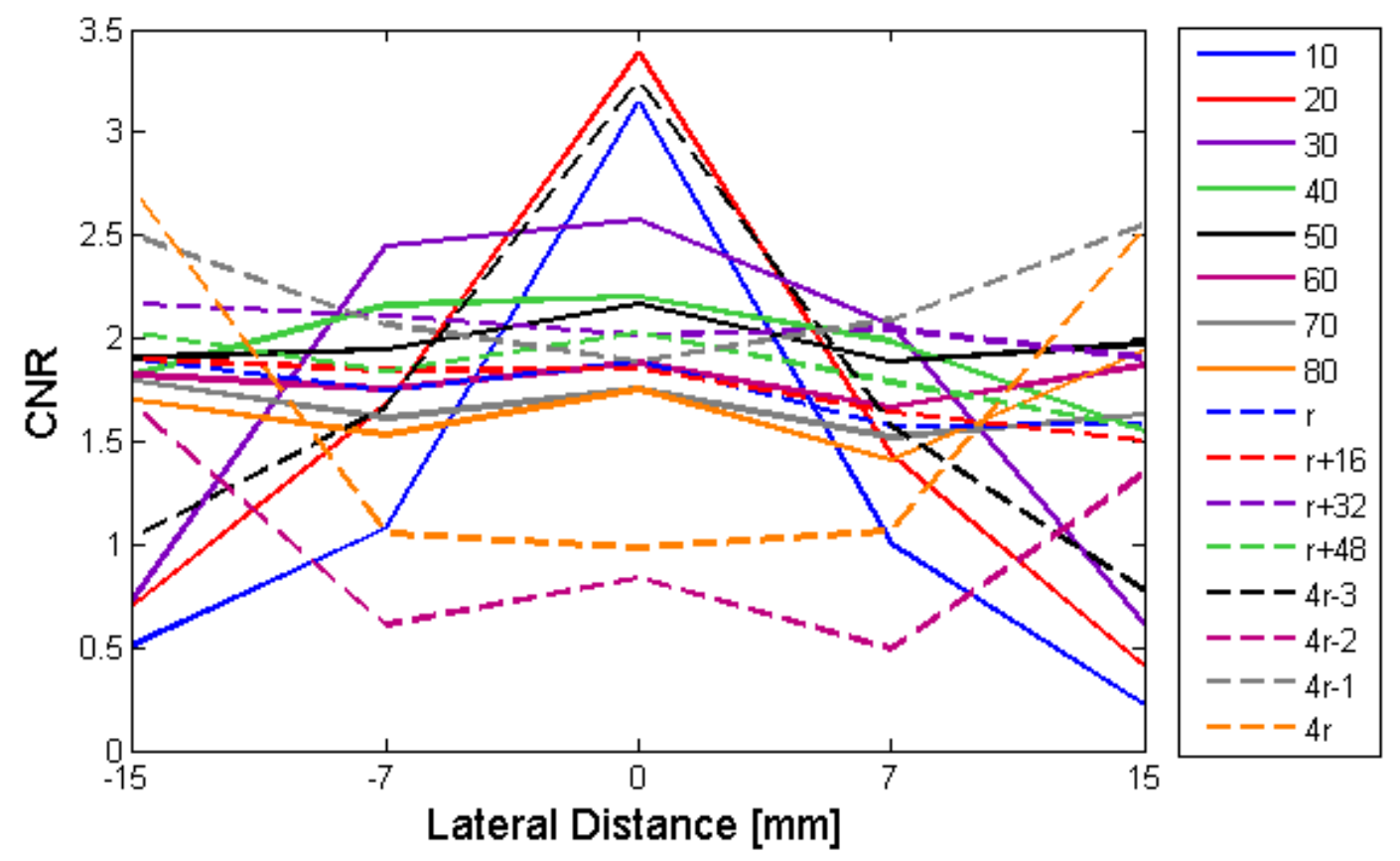

Fig. B.2. Contrast-to-noise value anechoic inclusion of SAPW of different range of transmission angle from \pm 10 degrees to \pm 80 degrees and different quarter Hadamard transmissions at different lateral location at depth $20 \mathrm{~mm}$. 


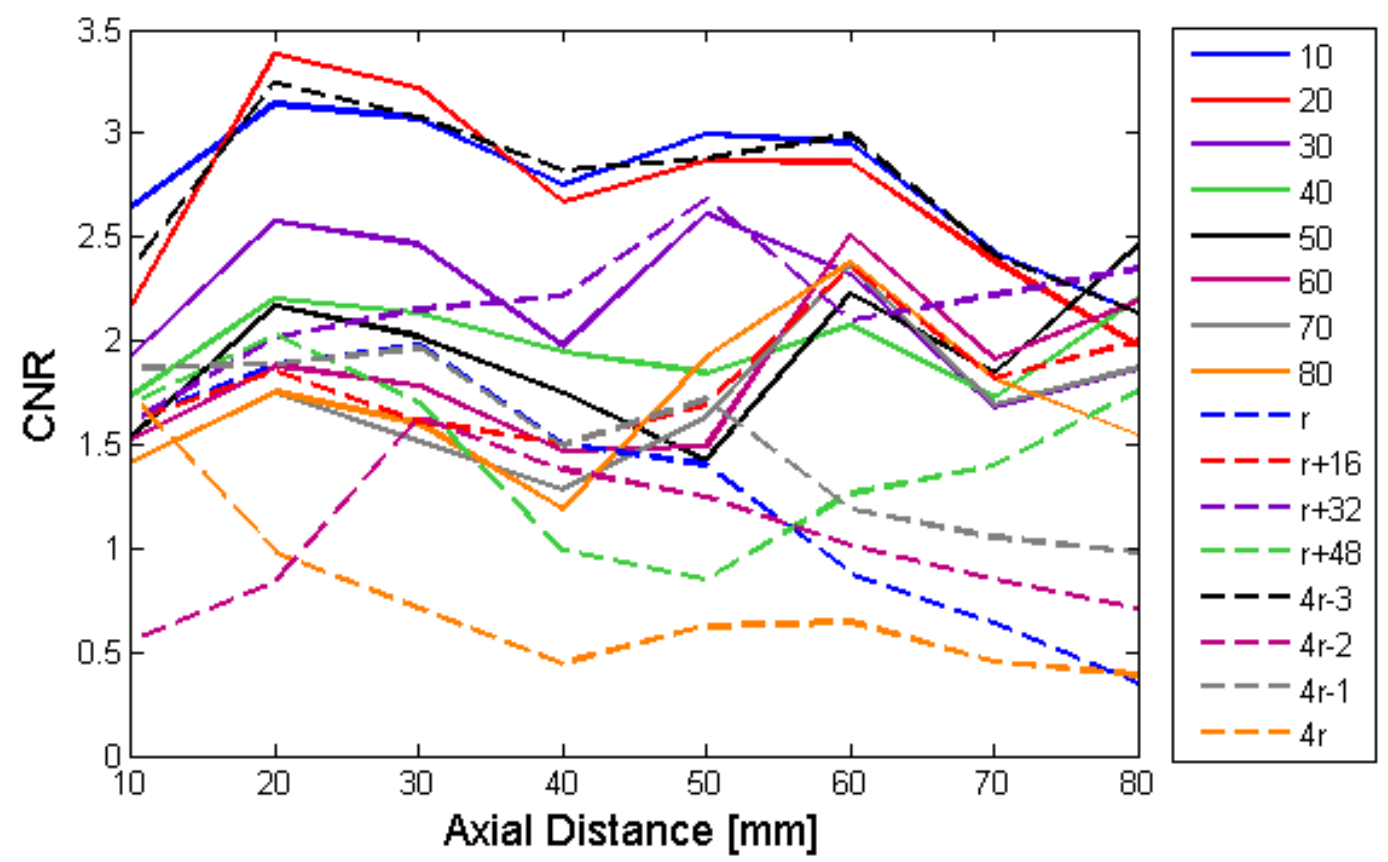

Fig. B.3. Contrast-to-noise value anechoic inclusion of SAPW of different range of transmission angle from \pm 10 degrees to \pm 80 degrees and different quarter Hadamard transmissions at different axial location at lateral distance $0 \mathrm{~mm}$.

These preliminary simulations demonstrate that different quarter Hadamard transmissions can be chosen depending on the region of the interest. This decoding method may be also applied to Bmode and any synthetic aperture sequential beamforming. Furthermore, the combination of the above transmissions can be used to improve the imaging quality. Figure B.4 shows the combination of \pm 20 degrees PW transmission and 4r transmission imaging since \pm 20 degrees PW transmission gives the better detection in the region under the aperture and 4r shows better detection of the region outside of the aperture. 

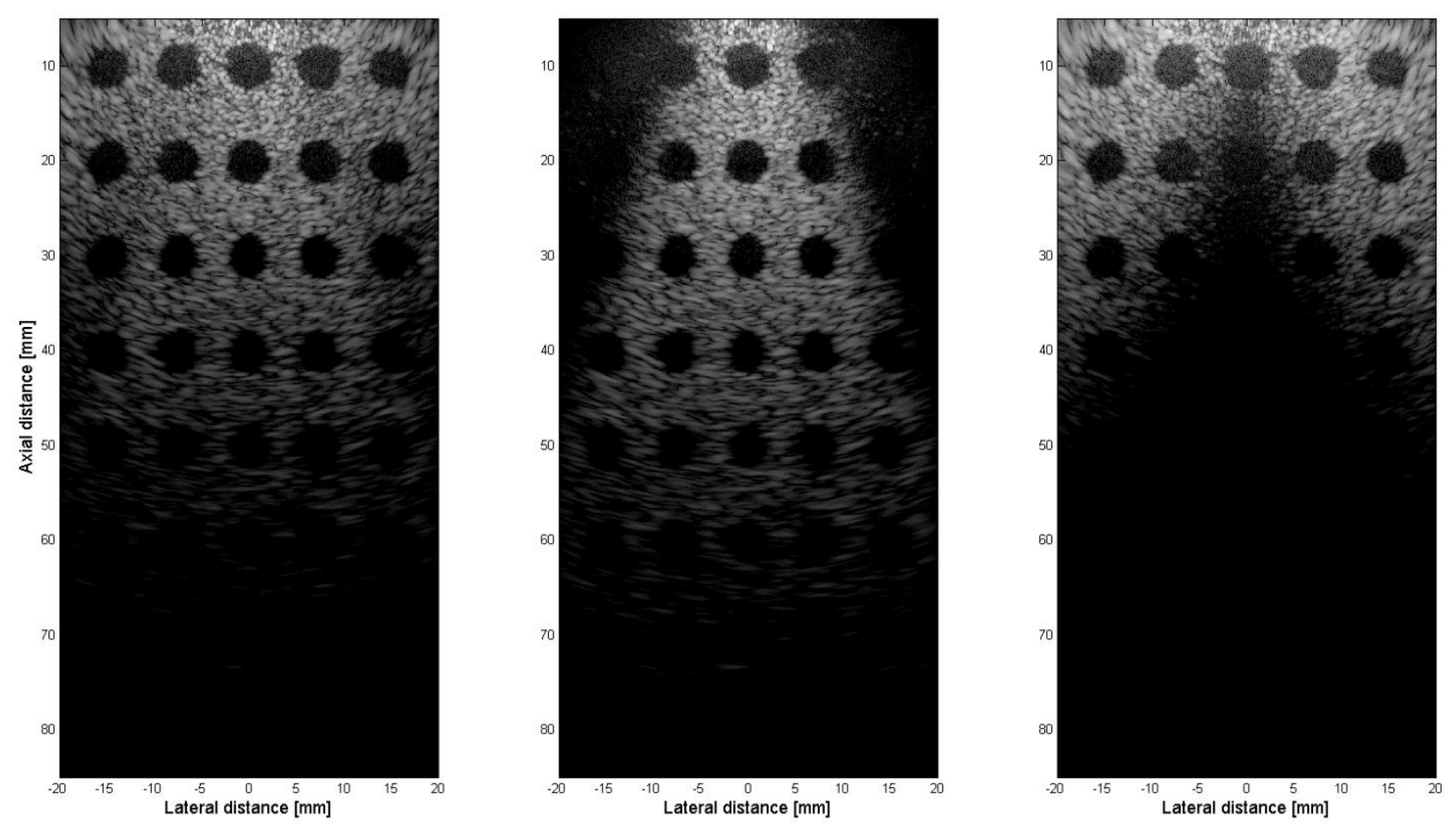

Fig. B.4. Simulated log-enveloped beamformed imaging of anechoic soft tissue phantom with combination of \pm 20 degrees SAPW and 4r quarter of Hadamard encoded transmissions (left), \pm 20 degrees SAPW only (middle) and 4r quarter of Hadamard encoded transmissions only (right). 


\section{REFERENCES FOR APPENDIX B}

[1] P. Gong, A. Moghimi, M. C. Kolios, and Y. Xu, "Delay-encoded Transmission in Synthetic Transmit Aperture (DE-STA) Imaging," IEEE International Ultrasonics Symposium (IUS), pp.1005-1008, 2014.

[2] P. Gong, M. C. Kolios, and Y. Xu, "Delay-encoded Transmission and Image Reconstruction Method in Synthetic Transmit Aperture Imaging," IEEE Trans. Ultrasonics, Ferroelectrics and Frequency Control, vol. 62, no. 10, Oct. 2015.

[3] P. Gong, M. C. Kolios, and Y. Xu, " Pseudo-Inverse (PI) Decoding process in Delay-encoded Synthetic Transmit Aperture (DE-STA) Imaging," IEEE Trans. Ultrasonics, Ferroelectrics and Frequency Control, in press, 2015.

[4] R. Y. Chiao, L. J. Thomas, and S. D. Silverstein, "Sparse array imaging with spatiallyencoded transmits," in Proc. IEEE Ultrason. Symp., pp. 1679-1682, 1997. 